\title{
A DNA damage signal activates and derepresses exon inclusion in Drosophila TAF1 alternative splicing
}

\author{
MATTHEW S. MARENGO and DAVID A. WASSARMAN \\ University of Wisconsin School of Medicine and Public Health, Department of Pharmacology, Molecular and Cellular Pharmacology Program, \\ Madison, WI 53706, USA
}

\begin{abstract}
Signal-dependent alternative splicing is important for regulating gene expression in eukaryotes, yet our understanding of how signals impact splicing mechanisms is limited. A model to address this issue is alternative splicing of Drosophila TAF1 pre-mRNA in response to camptothecin (CPT)-induced DNA damage signals. CPT treatment of Drosophila S2 cells causes increased inclusion of TAF1 alternative cassette exons 12a and 13a through an ATR signaling pathway. To evaluate the role of TAF1 premRNA sequences in the alternative splicing mechanism, we developed a TAF1 minigene (miniTAF1) and an S2 cell splicing assay that recapitulated key aspects of CPT-induced alternative splicing of endogenous TAF1. Analysis of miniTAF1 indicated that splice site strength underlies independent and distinct mechanisms that control exon 12a and 13a inclusion. Mutation of the exon 13a weak $5^{\prime}$ splice site or weak $3^{\prime}$ splice site to a consensus sequence was sufficient for constitutive exon 13a inclusion. In contrast, mutation of the exon 12a strong $5^{\prime}$ splice site or moderate $3^{\prime}$ splice site to a consensus sequence was only sufficient for constitutive exon 12a inclusion in the presence of CPT-induced signals. Analogous studies of the exon $133^{\prime}$ splice site suggest that exon 12a inclusion involves signal-dependent pairing between constitutive and alternative splice sites. Finally, intronic elements identified by evolutionary conservation were necessary for full repression of exon 12a inclusion or full activation of exon 13a inclusion and may be targets of CPT-induced signals. In summary, this work defines the role of sequence elements in the regulation of TAF1 alternative splicing in response to a DNA damage signal.
\end{abstract}

Keywords: S2 cells; Drosophila; TFIID; camptothecin; alternative splicing; abl

\section{INTRODUCTION}

Splicing is the process by which introns are removed from pre-messenger RNA (pre-mRNA) and exons are ligated together to form mature mRNA. Integral to this process is the spliceosome, which serves to distinguish introns from exons and to carry out the catalytic steps that result in intron removal and exon ligation. The spliceosome contains five small nuclear ribonucleoprotein (snRNP) complexes, $\mathrm{U} 1, \mathrm{U} 2, \mathrm{U} 4, \mathrm{U} 5$, and $\mathrm{U} 6$, and numerous protein factors (Brow 2002; Patel and Steitz 2003). Early recognition of the $5^{\prime}$ end of introns is achieved by base-pairing of sequences at the $5^{\prime}$ end of $U 1$ snRNA to $5^{\prime}$ splice site sequences that span the exon-intron boundary (Zhuang and Weiner 1986; Séraphin et al. 1988; Siliciano and Guthrie 1988). In

Reprint requests to: David A. Wassarman, University of Wisconsin School of Medicine and Public Health, Department of Pharmacology, 1300 University Avenue, Madison, WI 53706, USA; e-mail: dawassarman@ wisc.edu; fax: (608) 262-1257.

Article published online ahead of print. Article and publication date are at http://www.rnajournal.org/cgi/doi/10.1261/rna.1048808.
Drosophila melanogaster $5^{\prime}$ splice site sequences conform to the consensus $\mathrm{A}_{-2} \mathrm{G}_{-1} / \mathrm{G}_{+1} \mathrm{U}_{+2} \mathrm{R}_{+3} \mathrm{~A}_{+4} \mathrm{G}_{+5} \mathrm{U}_{+6}$ (exon/ intron, $\mathrm{R}=$ purine) (Weir and Rice 2004; Sheth et al. 2006). Later, $U 6$ snRNA replaces $U 1$ snRNA and base-pairs to $5^{\prime}$ splice site positions +2 to +6 (Wassarman and Steitz 1992; Kandels-Lewis and Séraphin 1993; Lesser and Guthrie 1993). Early recognition of the $3^{\prime}$ end of introns is achieved by the heterodimeric U2 snRNP auxiliary factor (U2AF) (Ruskin et al. 1988; Zamore and Green 1989; Zamore et al. 1992). The small subunit of U2AF recognizes the invariant $A G$ within the $3^{\prime}$ splice site consensus sequence $\mathrm{U}_{-6} \mathrm{U}_{-5} \mathrm{~N}_{-4} \mathrm{C}_{-3} \mathrm{~A}_{-2} \mathrm{G}_{-1} / \mathrm{R}_{+1}$ (intron/exon, $\mathrm{N}=$ any nucleotide) at the intron-exon boundary, and the large subunit of U2AF recognizes the polypyrimidine tract that precedes the 3' splice site (Zamore and Green 1989). Efficient binding of U1 snRNP or U2AF to $5^{\prime}$ or $3^{\prime}$ splice sites, respectively, recruits spliceosome components across the exon, in a process known as exon definition (Berget 1995).

In metazoan organisms, splicing mechanisms are complicated by the fact that most pre-mRNAs contain more than two exons, which affords combinatorial options for 
the ligation of $5^{\prime}$ and $3^{\prime}$ splice sites. This complexity underlies regulated alternative splicing, in which pre-mRNA sequences are differentially defined as exon or intron under different physiological conditions (Stamm 2002; Black 2003; Shin and Manley 2004; Schwerk and Schulze-Osthoff 2005). For example, in cassette exon alternative splicing, a sequence may be defined as an exon and included in the mature mRNA under one condition but defined as intronic and excluded from the mature mRNA under another condition. Regulated alternative splicing can lead to two distinct outcomes. It can generate mature mRNAs that encode functionally related but distinct proteins, or it can inhibit gene expression by generating mature mRNAs that include premature stop codons and are subject to nonsense-mediated decay (Cuccurese et al. 2005).

Pre-mRNA sequences and RNA binding proteins are important for defining which $5^{\prime}$ and $3^{\prime}$ splice sites will be used during alternative splicing (Graveley 2001). RNA binding proteins can activate or repress use of splice sites by binding exon sequences (exonic splicing enhancers [ESEs] or exonic splicing silencers [ESSs]) or intron sequences (intronic splicing enhancers [ISEs] or intronic splicing silencers [ISSs]). For example, SR protein family members commonly bind ESEs and stimulate utilization of $5^{\prime}$ and $3^{\prime}$ splice sites that border constitutive and alternative exons (Ram and Ast 2003). In contrast, hnRNP proteins commonly bind ESSs and antagonize the function of SR proteins (Rothrock et al. 2005).

An emerging theme in alternative splicing is that RNA binding proteins function to facilitate or inhibit binding of U1 snRNP or U2AF to alternative exon $5^{\prime}$ or $3^{\prime}$ splice sites, respectively, which statistically tend to be weaker than constitutive exon splice sites (Itoh et al. 2004). For example, in response to DNA damage, the RNA binding protein TIA-1 binds a U-rich ISE and facilitates binding of U1 snRNP to a weak $5^{\prime}$ splice site, which in turn enhances U2AF binding to the upstream $3^{\prime}$ splice site (Förch et al. 2002; Izquierdo et al. 2005). Consistent with this mechanism, competition assays revealed that the stability of the U1 snRNA-5' splice site duplex dictates the choice between two nearby $5^{\prime}$ splice sites, and knockdown of U2AF subunits inhibits weak $3^{\prime}$ splice site recognition, while overexpression of the large U2AF subunit is sufficient for weak 3' splice site recognition (Zhuang and Weiner 1986; Pacheco et al. 2006).

As a model to understand signal-dependent alternative splicing mechanisms, we have studied the regulation of $D$. melanogaster TAF1 pre-mRNA alternative splicing. The TAF1 gene encodes TAF1 (TBP-associated factor 1), a subunit of the general transcription factor TFIID (Weinzierl et al. 1993). We previously demonstrated that alternative splicing inclusion of two cassette exons, 12a and 13a, generates four TAF1 mRNA isoforms, TAF1-1, TAF1-2, TAF1-3, and TAF1-4 (Fig. 1; Katzenberger et al. 2006). Regulation of TAF1 alternative splicing is mediated by tissue-specific signals and DNA damage signals.

Double-stranded DNA breaks caused by ionizing radiation (IR) or the topoisomerase I inhibitor camptothecin (CPT) activates the ATM or ATR signaling pathway, respectively (Abraham 2001). Through an unknown mechanism, IR or CPT treatment of Drosophila S2 cells increases inclusion of both exons 12a and 13a and TAF1-4 levels
A

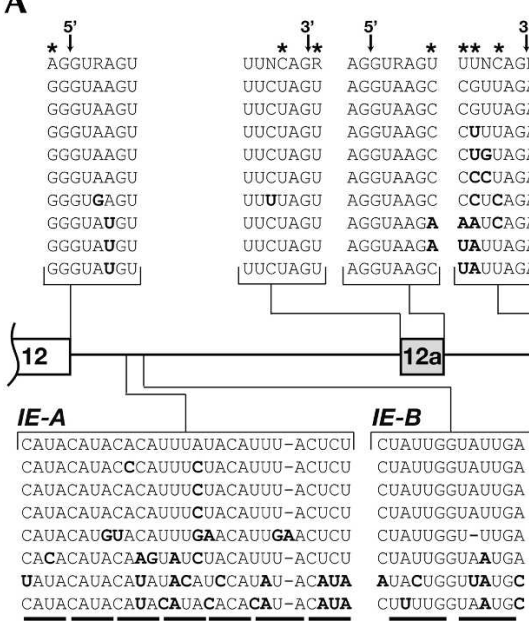

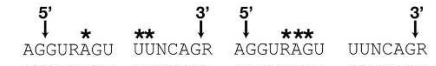
GGUGGGU AAGCAGA AGGUCUUU UUUCAGA AGGUGGGU AAGCAGA AGGUCUUU UUUCAGA AgGUGGgU AagCAGA AgGUCUUU UUUCAGA AGgugGgu gagCAGA AgGUCUCU UUUCAGA AgguggGU GagCAGA AgGUCUCU UUCCAGA aggugggu caAuaga agguUca uUCCag AGGUGGGU UAAUAGA AGGUUCAU UUUUCAGA GGUGGUU CAAUAGA

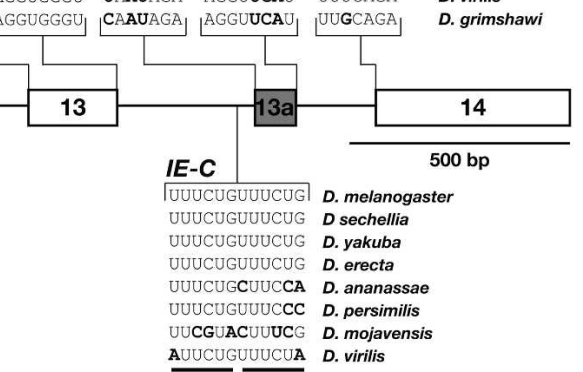

B

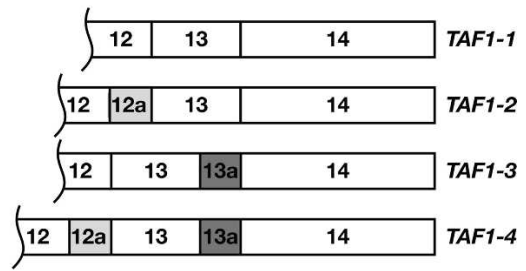

FIGURE 1. TAF1 alternative exons are flanked by evolutionarily conserved splice sites and intronic elements. $(A)$ A schematic diagram of the $D$. melanogaster TAF1 pre-mRNA with exons depicted as boxes and introns as lines. Above are sequences for 5' and 3' splice sites for Drosophila species arranged from least to most divergent (Schawaroch 2002). D. melanogaster consensus splice site sequences are shown at the top with asterisks $\left(^{*}\right)$ indicating positions in D. melanogaster TAF1 that differ from the consensus (Weir and Rice 2004; Sheth et al. 2006). Arrows indicate the site of cleavage by the spliceosome. Below are sequences of evolutionarily conserved intronic elements (IE) A-C. Nucleotides indicated in bold typeface differ from D. melanogaster. Underlines indicate repeated sequence within IEs. (B) Schematic diagrams of the $3^{\prime}$ end of TAF1 mRNA isoforms generated by alternative splicing (Katzenberger et al. 2006). 
(Katzenberger et al. 2006). Although the splicing effect of IR requires ATM and CHK2 and the splicing effect of CPT requires ATR and CHK1, either treatment up-regulates TAF1-4 levels, suggesting that the signaling pathways converge on a common set of splicing regulatory factors and that inclusion of exons $12 \mathrm{a}$ and $13 \mathrm{a}$ is coordinately controlled. However, the predominance of TAF1-2 and TAF1-3 mRNA isoforms in Drosophila testes and ovaries, respectively, suggests that exon $12 \mathrm{a}$ and $13 \mathrm{a}$ inclusion events can be regulated independently. Here, we present studies aimed at determining the extent to which TAF1 premRNA sequences dictate TAF1 alternative splicing patterns in response to CPT-induced DNA damage signals.

\section{RESULTS}

\section{The strength of splice sites associated with TAF1 exons $12 a$ and $13 a$ is substantially different}

To understand the importance of splice site sequences in TAF1 alternative splicing mechanisms, several criteria were used to evaluate the strength of the splice sites involved in alternative splicing events. First, the sequence of each splice site was compared to the D. melanogaster consensus sequence. The assumption was that consensus match frequency is directly proportional to splice site strength. Note that the D. melanogaster $5^{\prime}$ splice site consensus sequence is perfectly complementary to the U1 snRNA $5^{\prime}$ end. By this measure, the strongest splice sites were $5^{\prime}$ splice sites associated with exons $12,12 \mathrm{a}$, and 13 and the $3^{\prime}$ splice site associated with exon 14 (Table 1). The weakest splice sites were the $3^{\prime}$ splice site associated with exon 13 and the $5^{\prime}$ splice site associated with exon 13 a.

Second, an algorithm developed by Freund et al. (2003) was used to evaluate the hydrogen bonding potential between $5^{\prime}$ splice sites and the U1 snRNA $5^{\prime}$ end. By this measure, $5^{\prime}$ splice sites associated with exons 12, 12a, and

TABLE 1. TAF1 pre-mRNA splice site strength

\begin{tabular}{lcccl}
\hline Exon splice site & Consensus $(\%)^{\mathrm{a}}$ & H-bond & Frequency $(\%)^{\mathrm{c}}$ & Strength \\
\hline $125^{\prime}$ & 88 & $17(\mathrm{HC})$ & 75.2 & Strong \\
$12 \mathrm{a} 3^{\prime}$ & 71 & - & 7.2 & Moderate \\
$12 \mathrm{a} 5^{\prime}$ & 88 & $17(\mathrm{HC})$ & 41.5 & Strong \\
$133^{\prime}$ & 57 & - & 1.2 & Weak \\
$135^{\prime}$ & 88 & $21(\mathrm{HC})$ & 51.4 & Strong \\
$13 \mathrm{a} 3^{\prime}$ & 71 & - & 1.2 & Weak \\
$13 \mathrm{a} 5^{\prime}$ & 63 & $10(\mathrm{LC})$ & 0.2 & Weak \\
$143^{\prime}$ & 100 & - & 100.0 & Strong \\
\hline
\end{tabular}

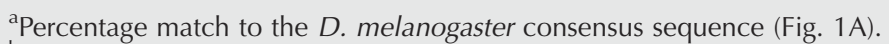

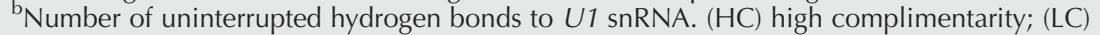
low complimentarity (Freund et al. 2003). The consensus 5' splice site mutants have H-bond scores of 23.

${ }^{\mathrm{C}}$ Frequency of match to 42,629 splice sites in a D. melanogaster splice site database, normalized to the 1464 matches for exon $143^{\prime}$ splice site (Sheth et al. 2006).
13 were in the high complementary (i.e., strong) class, while the $5^{\prime}$ splice site associated with exon 13a was in the low complementarity (i.e., weak) class (Table 1 ).

Finally, we determined how many of the 42,629 documented splice sites in D. melanogaster were identical to TAF1 splice sites involved in alternative splicing (Sheth et al. 2006). The assumption was that frequency is directly proportional to splice site strength. By this measure, the strongest splice sites were the $5^{\prime}$ splice site associated with exon 12 and the $3^{\prime}$ splice site associated with exon 14 (Table 1). The weakest splice sites were the $3^{\prime}$ splice sites associated with exons 13 and $13 \mathrm{a}$ and the $5^{\prime}$ splice site associated with exon 13a.

Taking all three measures into account, splice site strengths were categorized as weak, moderate, or strong. As expected, splice sites associated with constitutive exons 12 and 14 were strong and splice sites associated with alternative exon 13a were weak. Unexpectedly, the 3' splice site associated with constitutive exon 13 was weak and the splice sites associated with alternative exon 12 a were moderate or strong. Thus, the strength of splice sites associated with exons $12 \mathrm{a}$ and 13 a substantially differs as does the strength of constitutive splice sites that pair with exon 12a or 13a splice sites. These differences suggest that the mechanisms underlying signal-dependent exon 12a and 13a inclusion are fundamentally dissimilar.

\section{The inferred strength of TAF1 splice sites is evolutionarily conserved}

To gauge the importance of splice site strength differences observed in D. melanogaster TAF1 for the regulation of TAF1 alternative splicing, we compared TAF1 gene sequences among evolutionarily divergent Drosophila species. The biological relevance of this comparison is supported by studies of TAF1 expression in D. virilis, which diverged from $D$. melanogaster $\sim 40$ million years ago (Schawaroch 2002). Sequence analysis of TAF1 cDNAs revealed that $D$. virilis TAF1 splice junctions were identical to $D$. melanogaster (P. Vu, M.S. Marengo, and D.A. Wassarman, unpubl. observations). In addition, the relative ratio of TAF1 mRNA isoforms in D. virilis tissues was similar to that in D. melanogaster, suggesting that the signaling pathways that regulate TAF1 alternative splicing are evolutionarily conserved.

Analysis of the TAF1 alternatively spliced region that spans exons 12-14 revealed that both consensus and nonconsensus bases within splice sites were highly conserved among all species examined (Fig. 1A). In almost all cases where sequence divergence occurred, 
the altered bases occurred at positions that were variable in the $D$. melanogaster consensus sequence or nonconsensus bases were changed to other nonconsensus bases. To illustrate, for the exon 13 a $5^{\prime}$ splice site, differences among the species occurred at positions $+3,+4$, and +5 , but in each case nonconsensus bases were changed to other nonconsensus bases. The exon $133^{\prime}$ splice site was an exception to this mode of conservation. The sequence of the exon $133^{\prime}$ splice site was highly divergent. Both consensus and nonconsensus sequences occurred at positions $-6,-5$, and -3 ; however, at least two nonconsensus nucleotides were maintained in each species. Thus, strong splice sites that differ in sequence and weak splice sites that differ in sequence are evolutionarily conserved, suggesting that the arrangement of weak, moderate, and strong splice sites is important to the TAF1 alternative splicing mechanism.

\section{Evolutionary comparison identifies potential intronic splicing regulatory elements}

To identify non-splice-site sequence elements involved in the regulation of TAF1 alternative splicing, we searched for sequences that were conserved among Drosophila species. Exons 12-14 were highly conserved, but this may be to conserve encoded protein sequence. In contrast, most of the intron sequences were highly diverged, except for three small regions, intronic elements (IE) $A-C$ (Fig. 1A). All of the conserved elements were pyrimidine rich and contained repeated sequences ( $I E-A$, CAUA; $I E-B$, UAUUG; and $I E-C$, UUUCUG). Repetitive sequences are a feature of some splicing regulatory elements (Black 2003). Thus, we hypothesized that these elements serve as binding sites for signal-regulated RNA binding proteins that regulate TAF1 cassette exon inclusion.

\section{A TAF1 minigene system recapitulates CPT- but not IR-induced up-regulation of TAF1-4 splicing}

To determine the importance of splice site strength and conserved intron elements for signal-dependent TAF1 alternative splicing, we developed a system for analyzing splicing of a TAF1 minigene (miniTAF1) in Drosophila S2 cells (Fig. 2A). In the construction of miniTAF1, an effort was made to include all of the sequences that could influence the splicing mechanism. In particular, since there is compelling evidence that promoter structure influences alternative splicing patterns, the endogenous TAF1 promoter (a 2.6-kb region upstream of the transcription start site) was used to drive expression of miniTAF1 (Goldstrohm et al. 2001; Kornblihtt 2005). In addition to the TAF1 promoter, miniTAF1 contained sequences encoding a FLAGepitope tag at the amino terminus, exons $12-14$ and the intervening introns, and the endogenous TAF1 3' region (a $1-\mathrm{kb}$ region downstream from the translation stop codon).
A

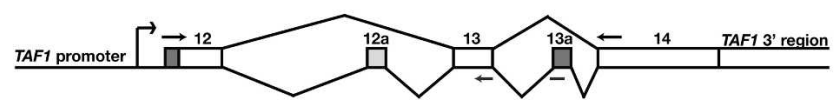

B
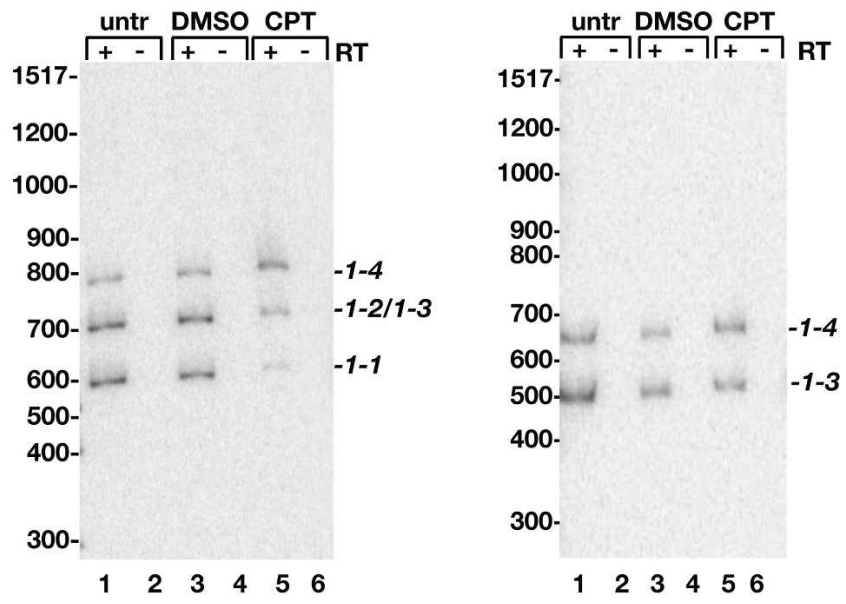

C

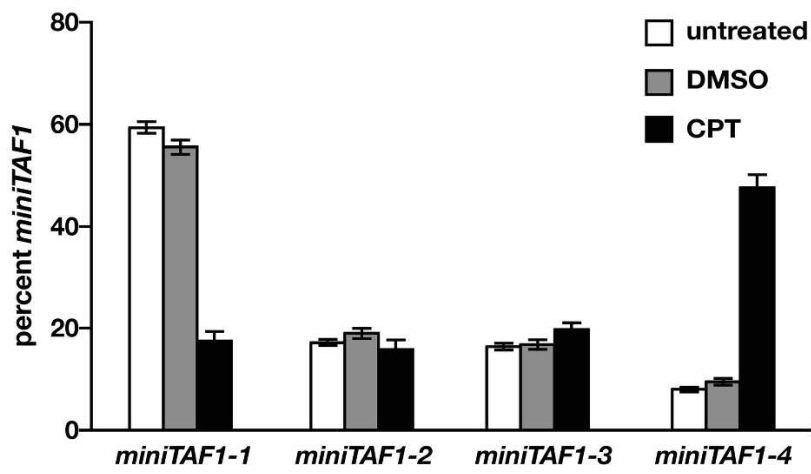

FIGURE 2. MiniTAF1 recapitulates CPT-induced up-regulation of TAF1-4 splicing. (A) A schematic diagram of the miniTAF1 plasmid. Boxes indicate exons and arrows indicate the position and direction of primers used for RT-PCR to quantitate expression of miniTAF1 mRNA isoforms. The exon 13/13a arrow is broken because the primer was complementary to the exon-exon junction. (B) Analysis of miniTAF1 expression by RT-PCR. On the left is analysis of all four mRNA isoforms, and on the right is analysis of exon 13a-containing mRNA isoforms. Samples were analyzed from untreated (untr) S2 cells (lanes 1,2), DMSO-treated S2 cells (lanes 3,4), or CPT-treated S2 cells (lanes 5,6). Even numbered lanes contained RT-PCR samples in which reverse transcriptase (RT) was left out as a control for plasmid contamination. Positions of DNA size standards in base pairs are indicated to the left of each panel, and positions of miniTAF1 isoforms are indicated to the right of each panel. $(C)$ Quantitation of the effect of CPT treatment on miniTAF1 mRNA isoforms $(n=18)$. These data are pooled from experiments in panel $B$ and Figures 3-10. Error bars represent the standard errors of the mean.

Drosophila S2 cells were transfected with miniTAF1 plasmid, and radioactive RT-PCR was used to detect and quantitate miniTAF1 mRNA isoform levels. A primer complementary to sequences encoding the FLAG-epitope tag in combination with a primer complementary to exon 14 was 
used to detect all four miniTAF1 mRNA isoforms in a single reaction (Fig. 2). Cloning and sequencing were used to identify reaction products (data not shown). Because miniTAF1-2 and miniTAF1-3 differed by only $6 \mathrm{nt}$, they comigrated upon gel electrophoresis, and a second, exon 13aspecific RT-PCR was used so that all four miniTAF1 mRNA isoforms could be quantitated.

To determine the extent to which the mechanism of CPT-induced TAF1 alternative splicing was mimicked by miniTAF1, we examined miniTAF1 splicing in S2 cells in the absence or presence of CPT. Analysis $36 \mathrm{~h}$ posttransfection revealed that the steady state level of miniTAF1 mRNA isoforms was similar to endogenous TAF1 mRNA isoforms, with TAF1-1 $>$ TAF1-2 $\cong$ TAF1-3 $>$ TAF1-4 (Fig. 2B,C). CPT treatment of miniTAF1-transfected cells resulted in significant down-regulation of miniTAF1-1 (Bonferroni $t=20, P<0.001$ ) and significant up-regulation of miniTAF1-4 (Bonferroni $t=18, P<0.001$ ), as observed for endogenous TAF1-4 (Katzenberger et al. 2006). These results indicated that the miniTAF1 system could be used to identify pre-mRNA elements necessary for CPT-induced TAF1 alternative splicing.

To determine the extent to which the mechanism of IRinduced TAF1 alternative splicing was mimicked by miniTAF1, we examined miniTAF1 splicing in S2 cells in the absence or presence of IR. Analysis $3 \mathrm{~h}$ after IR treatment revealed a modest up-regulation of endogenous TAF1-4 (one-tailed $t=2.8, P=0.055$ ) but no change in miniTAF1-4 (Fig. 3). Thus, miniTAF1 contains sequence elements necessary for CPT-induced miniTAF1-4 splicing but is missing elements essential for IR-induced miniTAF1-4 splicing.

\section{A consensus TAF1 exon $13 a 5^{\prime}$ splice site is sufficient for constitutive exon 13a inclusion}

The weak exon 13a 5 ' splice site was an attractive potential source of alternative splicing regulation (Fig. 1A; Table 1). To determine the extent to which binding of the $U 1$ snRNA to the exon 13 a $5^{\prime}$ splice site specifies exon 13 a inclusion, we mutated the splice site in miniTAF1 to match the consensus sequence ( $13 a 5^{\prime}$ cons mut) (Fig. 4A). Cells transfected with $13 a 5^{\prime}$ cons mut expressed significantly less miniTAF1-1 $(F=260, P<0.0001)$ and miniTAF1-2 $(F=$ 9.9, $P=0.02)$ and more miniTAF1-3 $(F=55, P=0.0003)$ and miniTAF1-4 $(F=170, P<0.0001)$ than those transfected with wild type ( $w t)$ miniTAF1 (Fig. 4B,C). In fact, $\sim 100 \%$ of $13 a 5^{\prime}$ cons mut mRNAs contained exon $13 \mathrm{a}$ compared to $\sim 30 \%$ for $w t$ mRNAs. Thus, even in the context of a weak exon 13 a $3^{\prime}$ splice site, a strong exon $13 \mathrm{a}$ $5^{\prime}$ splice site is sufficient for constitutive exon 13a inclusion.

Interactions between CPT and splicing were also examined. CPT treatment significantly down-regulated $w t$ but not $13 a 5^{\prime}$ cons mut miniTAF1-1 (Bonferroni $t=5.7, P<0.01$ and Bonferroni $t=0.093, P>0.05$, respectively), indicating that U1 snRNA binding bypasses the CPT-induced mechanism of
A
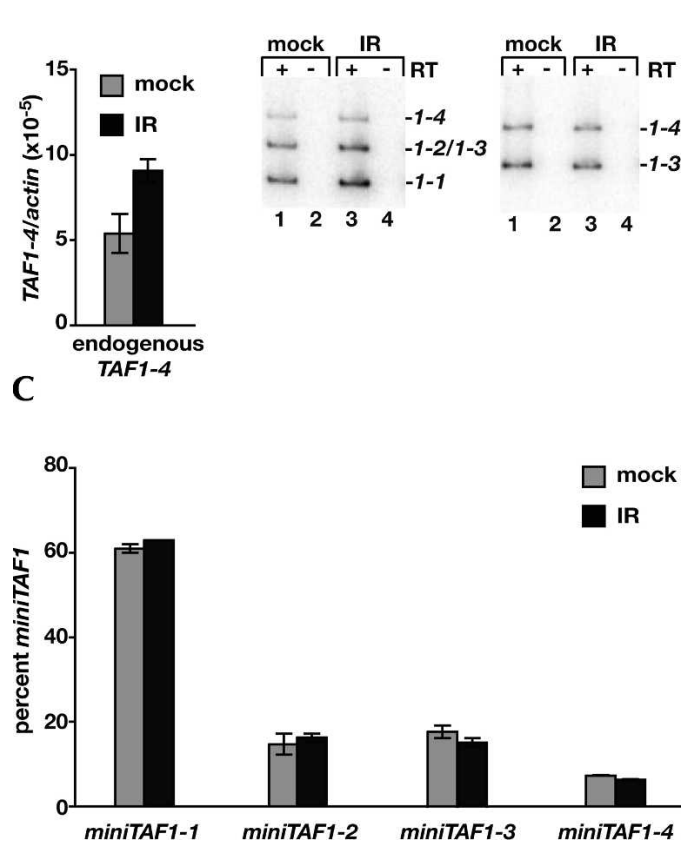

FIGURE 3. MiniTAF1 does not recapitulate IR-induced up-regulation of TAF1-4 splicing. (A) qPCR analysis of endogenous TAF1 mRNA levels from mock- or IR-treated cells. Error bars represent the standard errors of the mean. (B) Analysis of miniTAF1 expression by RT-PCR. On the left is analysis of all four mRNA isoforms, and on the right is analysis of exon 13a-containing mRNA isoforms. Samples were analyzed from mock-treated (mock) S2 cells (lanes 1,2) or IRtreated S2 cells (lanes 3,4). Even numbered lanes contained RT-PCR samples in which reverse transcriptase (RT) was left out as a control for plasmid contamination. Positions of miniTAF1 isoforms are indicated to the right of each panel. (C) Quantitation of the effect of IR treatment on miniTAF1 mRNA isoforms $(n=2)$.

exon 13a inclusion (Fig. 4B,C). CPT treatment also significantly up-regulated both $w t$ and $13 a 5^{\prime}$ cons mut miniTAF1-4 (Bonferroni $t=6.1, P<0.01$ and Bonferroni $t=6.0, P<0.01$, respectively), as compared to untreated cells. These data indicate that constitutive exon 13a inclusion does not affect the CPT-induced exon 12a inclusion mechanism (Fig. 4D).

\section{U1 snRNA binding to the TAF1 exon 13a 5 ' splice site is sufficient for constitutive exon $13 \mathrm{a}$ inclusion}

To further examine if efficient $U 1$ snRNA binding is sufficient to cause exon 13a inclusion, we generated a $U 1$ snRNA gene (mut U1) that had a $5^{\prime}$ end perfectly complementary to the endogenous TAF1 exon 13a $5^{\prime}$ splice site (Fig. 4A). Cells transfected with mut $U 1$ up-regulated endogenous TAF1-3 $(t=7.2, P=0.0055)$ and TAF1-4 mRNA $(t=5.2, P=0.0141)$, as measured by qPCR (Fig. $4 \mathrm{E}$ ). Note that these cells were not transfected with miniTAF1. Total TAF1 levels, as measured by qPCR of a region common to all four TAF1 mRNA isoforms, were not affected by mut $U 1(F=2.8, P=0.17)$. Also, transfection 
A

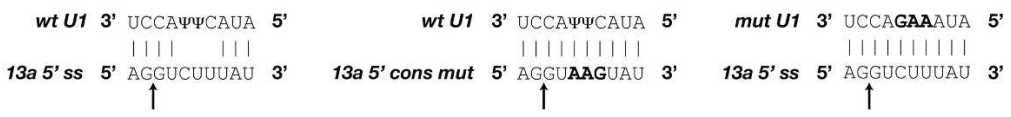

B

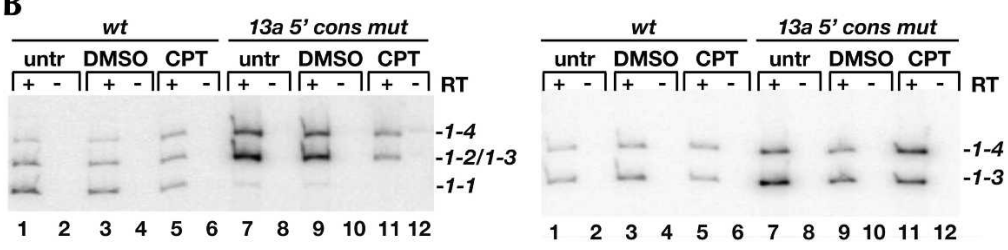

C

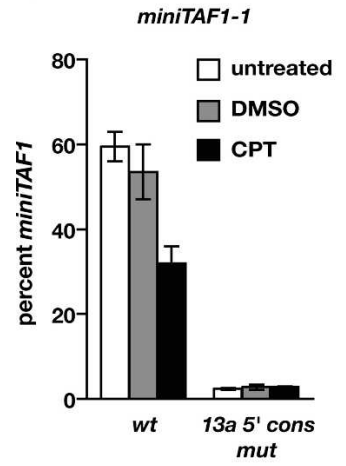

D
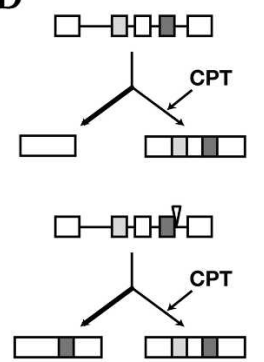

miniTAF1-3

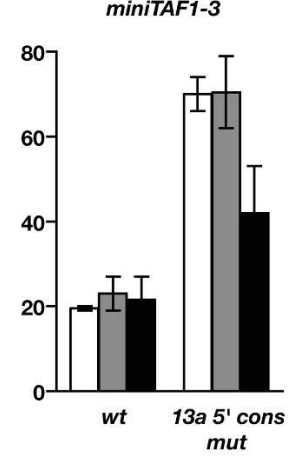

$\mathbf{E}$

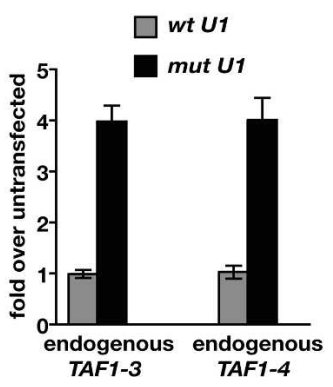

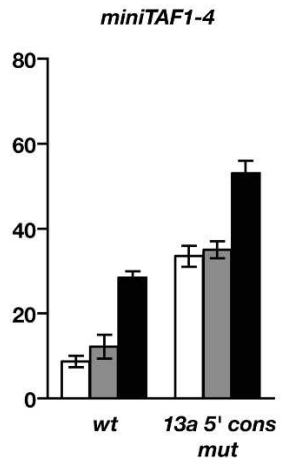

mut

FIGURE 4. Investigation of the role of the TAF1 exon 13a $5^{\prime}$ splice site in CPT-induced alternative splicing. (A) Shown is base pairing between the exon $13 \mathrm{a} 5^{\prime}$ splice site (ss) in miniTAF1 pre-mRNAs and U1 snRNAs. Expected base pairs are depicted as vertical lines. Pseudouridines in U1 snRNA are depicted as $\psi$. Arrows indicate splice sites and bold typeface indicates mutated nucleotides. (B) Analysis of miniTAF1 expression by RT-PCR. On the left is analysis of all four mRNA isoforms, and on the right is analysis of exon 13a-containing mRNA isoforms. Samples were analyzed from untreated (untr) S2 cells (lanes 1,2,7,8), DMSO-treated S2 cells (lanes 3,4,9,10), or CPT-treated S2 cells (lanes 5,6,11,12). Even numbered lanes contained RT-PCR samples in which reverse transcriptase (RT) was left out as a control for plasmid contamination. The transfected plasmid is indicated at the top. Positions of the miniTAF1 mRNA isoforms are shown to the right of each panel. (C) Quantitation of miniTAF1 mRNA isoforms from panel $B(n=2)$. Error bars represent the standard errors of the mean. (D) A summary of the effect of CPT-induced signaling on miniTAF1 alternative splicing in the context of the consensus exon 13 a $5^{\prime}$ splice site $\left(13 a 5^{\prime}\right.$ cons mut). Shaded boxes indicate alternative exons 12a and 13a. The open arrowhead indicates the mutated exon 13a $5^{\prime}$ splice site. (E) qPCR analysis of endogenous TAF1 mRNA levels from cells transfected with $w t$ U1 snRNA plasmid or mut U1 snRNA plasmid. Error bars represent the standard errors of the mean.

of a wild-type $U 1$ snRNA gene ( $w t U 1$ ) did not affect TAF1 isoform levels. Since the transfection efficiency was $\sim 50 \%$ (as estimated by GFP cotransfection), up-regulation of TAF1-3 and TAF1-4 splicing was even higher than the population measurement revealed (data not shown). Thus,

consistent with the miniTAF1 studies, these data indicate that binding of the $U 1$ snRNA is likely to play a major role in the mechanism of signal-induced exon 13a inclusion.

\section{A consensus TAF1 exon 13a 3' splice site is sufficient for constitutive exon 13a inclusion}

Similar to the weak $5^{\prime}$ splice site, the weak exon 13a $3^{\prime}$ splice site was an attractive potential source of alternative splicing regulation (Fig. 1A; Table 1). To examine this possibility, we mutated the exon 13a $3^{\prime}$ splice site in miniTAF1 to match the $D$. melanogaster consensus sequence ( $13 a 3^{\prime}$ cons mut) (Fig. 5A). Cells transfected with $13 a 3^{\prime}$ cons mut expressed significantly less miniTAF1-1 $(F=5200, P<0.0001)$ and miniTAF1-2 $(F=540, P<0.0001)$ and more miniTAF1-3 $(F=6200, P<0.0001)$ and miniTAF1-4 $(F=1500, P<0.0001)$ than those transfected with wt miniTAF1 (Fig. 5B-D). Approximately $100 \%$ of $13 a 3^{\prime}$ cons mut transcripts contained exon 13a compared to $\sim 25 \%$ for $w t$ transcripts. CPT treatment significantly down-regulated $w t$ but not 13a 3' cons mut miniTAF1-1 (Bonferroni $t=40, P<0.001$ and Bonferroni $t=1.1, P>0.05$, respectively), and significantly up-regulated both $w t$ and $13 a 3^{\prime}$ cons mut miniTAF1-4 (Bonferroni $t=35, P<0.001$ and Bonferroni $t=26, P<0.001$, respectively), as compared to untreated cells. These data provide additional evidence that CPTinduced inclusion of exon 12a occurs independently of exon 13a inclusion and that, even in the context of a weak exon 13 a $5^{\prime}$ splice site, a strong exon 13 a $3^{\prime}$ splice site is sufficient for exon 13a inclusion.

\section{IE-C is an ISE for TAF1 exon 13a inclusion}

To further explore the regulation of signal-dependent exon 13a inclusion, we examined $I E-C$ because of its close proximity to exon 13a (26 nt upstream of the $3^{\prime}$ splice site) and its structure (two direct repeats of the sequence UUUCUG) (Fig. 1A). Splicing of miniTAF1 that contained mutations of evolutionarily conserved 
A

B
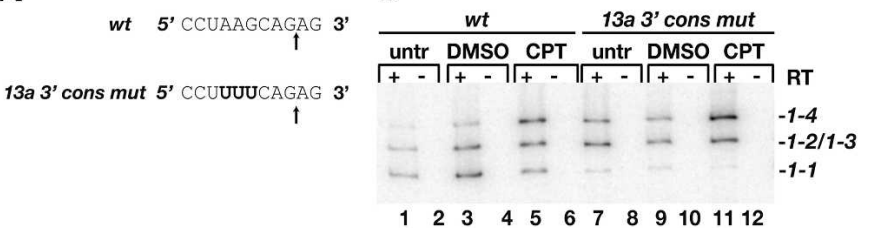

C
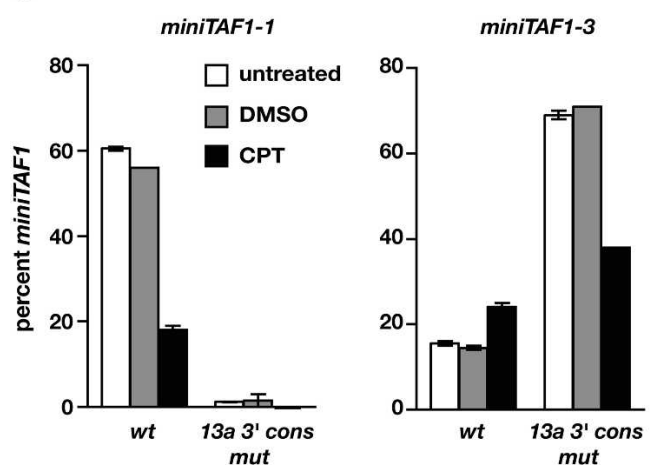

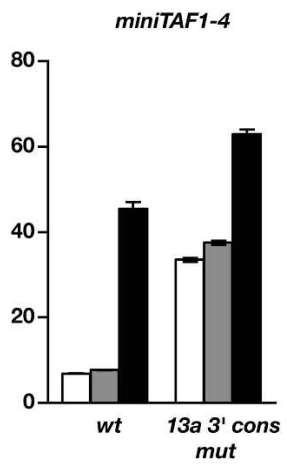

D

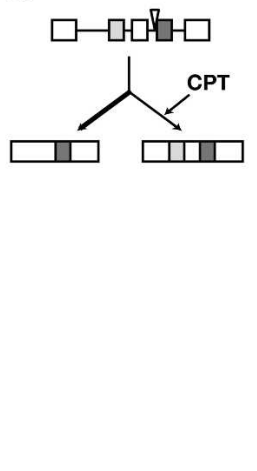

FIGURE 5. Investigation of the role of the TAF1 exon 13a 3 ' splice site in CPT-induced alternative splicing. (A) A comparison of wt and mutant (13a 3' cons mut) exon 13a 3' splice site sequences. Arrows indicate splice sites and bold typeface indicates mutated nucleotides. (B) Analysis of miniTAF1 expression by RT-PCR. On the left is analysis of all four mRNA isoforms, and on the right is analysis of exon 13a-containing mRNA isoforms. Samples were analyzed from untreated (untr) S2 cells (lanes 1,2,7,8), DMSO-treated S2 cells (lanes 3,4,9,10), or CPT-treated S2 cells (lanes 5,6,11,12). Even numbered lanes contained RT-PCR samples in which reverse transcriptase (RT) was left out as a control for plasmid contamination. The transfected plasmid is indicated at the top. Positions of the miniTAF1 mRNA isoforms are shown to the right of each panel. $(C)$ Quantitation of miniTAF1 isoforms from panel $B(n=2)$. Error bars represent the standard errors of the mean. $(D)$ A summary of the effect of CPT-induced signaling on miniTAF1 alternative splicing in the context of the consensus exon 13a 3' splice site (13a 3' cons mut). Shaded boxes indicate alternative exons $12 \mathrm{a}$ and 13a. The open arrowhead indicates the mutated exon 13a $3^{\prime}$ splice site.

uridines to adenosines in both half-sites (IE-C mut) was examined using the S2 cell assay (Fig. 6A). This study revealed that $\mathrm{CPT}$ treatment did not affect expression of $w t$ miniTAF1-2 (Bonferroni $t=1.5, P>0.05$ ); however, it significantly up-regulated expression of IE-C mut miniTAF1-2 (Bonferroni $t=7.5, P<0.001$ ), as compared to untreated cells (Fig. 6B-D). In addition, under all treatment conditions, cells transfected with IE-C mut expressed significantly less miniTAF1-4 relative to cells transfected with $w t$ miniTAF1 $(F=41, P<0.0001)$. Thus, in the context of IE-C mut, compared to wt miniTAF1, CPT treatment further up-regulated miniTAF1-2 but upregulated miniTAF1-4 to a lesser extent, suggesting that IE- $C$ functions as a CPT-regulated ISE that promotes exon 13a inclusion.

\section{IE- $C$ may be a general CPT-regulated splicing element}

To assess how generally IE-C functions as a CPT-regulated splicing element, we conducted a computational search for the IE-C repeat sequence in the D. melanogaster genome. This search identified $>10$ genes with $I E$ - $C$-like sequences located in close proximity to a $3^{\prime}$ splice site (data not shown). One of these genes, $a b l$, encodes the Drosophila homolog of the Abelson proto-oncogene (Henkemeyer et al. 1988). In the $a b l$ pre-mRNA, three copies of the $I E-C$ repeat sequence are juxtaposed to the $3^{\prime}$ splice site of an alternatively spliced 54-nt exon (UUUCUGUUUUUUCUGUUUCUGCAG/G, underline $=I E-C$ repeats, $/=$ intron-exon junction $)$. RTPCR analysis of endogenous abl mRNA isoforms revealed that inclusion of the alternative exon was up-regulated by CPT treatment of S2 cells (Fig. 6E). Cloning and sequencing were used to identify the reaction products (data not shown). Interestingly, alternative splicing of $a b l$ in response to CPT may be related to a function described for human $\mathrm{c}-\mathrm{Abl}$ in the modulation of CPT-induced apoptosis (Truong et al. 2003). Thus, IE-C may be a CPT-induced DNA damage-responsive element that coordinately controls alternative splicing of many Drosophila pre-mRNAs.

\section{A consensus TAF1 exon 12a $5^{\prime}$ splice site does not fully bypass CPT-induced exon 12a inclusion}

To examine the generality of the alternative splicing mechanism inferred from analysis of TAF1 exon 13a, we examined the TAF1 exon 12a $5^{\prime}$ splice site. Similar to exon 13a, exon 12a inclusion is induced by CPT; however, unlike the exon 13 a $5^{\prime}$ splice site that is weak, the exon $12 \mathrm{a} 5^{\prime}$ splice site is strong (Table 1). We examined the role of $U 1$ snRNA binding in exon 12a inclusion by mutating the $5^{\prime}$ splice site in miniTAF1 to match the D. melanogaster consensus sequence ( $12 a 5^{\prime}$ cons mut) (Fig. 7A). Cells transfected with $12 a 5^{\prime}$ cons mut expressed significantly less 


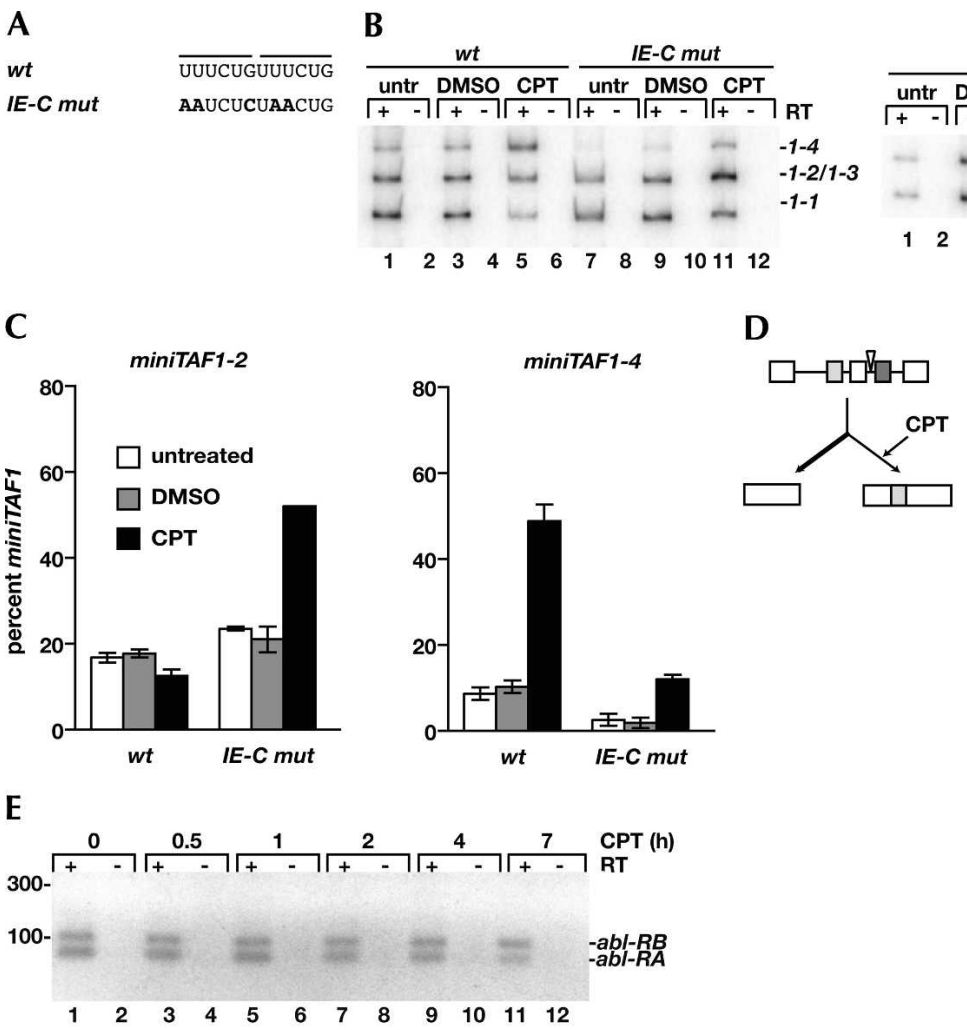

FIGURE 6. Investigation of the role of $I E-C$ in CPT-induced alternative splicing. (A) A comparison of $w t$ and mutant $I E-C$ (IE-C mut) sequences. Mutated nucleotides are indicated in bold typeface. Overlines indicate the tandem repeat sequence. (B) Analysis of miniTAF1 expression by RTPCR. On the left is analysis of all four mRNA isoforms, and on the right is analysis of exon 13a-containing mRNA isoforms. Samples were analyzed from untreated (untr) S2 cells (lanes 1,2,7,8), DMSO-treated S2 cells (lanes 3,4,9,10), or CPT-treated S2 cells (lanes 5,6,11,12). Even numbered lanes contained RT-PCR samples in which reverse transcriptase (RT) was left out as a control for plasmid contamination. The transfected plasmid is indicated at the top. Positions of the miniTAF1 mRNA isoforms are shown to the right of each panel. (C) Quantitation of miniTAF1 mRNA isoforms from panel $B(n=2-4)$. Error bars represent the standard errors of the mean. $(D)$ A summary of the effect of CPTinduced signaling on miniTAF1 alternative splicing in the context of the IE-C mutants. Shaded boxes indicate alternative exons 12a and 13a. The open arrowhead indicates the mutated IE-C. (E) RT-PCR analysis of $a b l$ mRNA isoform expression in CPT-treated S2 cells. Even numbered lanes contained RT-PCR samples in which RT was left out. abl-RB differs from abl-RA by the inclusion of a 54-nt alternative cassette exon (Henkemeyer et al. 1988).

miniTAF1-1 $(F=740, P<0.0001)$ and miniTAF1-3 $(F=$ 220, $P<0.0001)$ but more miniTAF1-2 $(F=280, P<$ $0.0001)$ and miniTAF1-4 $(F=440, P<0.0001)$ than those transfected with wt miniTAF1 (Fig. 7B-D). CPT treatment significantly down-regulated both $w t$ and $12 a 5^{\prime}$ cons mut miniTAF1-1 (Bonferroni $t=29, P<0.001$ and Bonferroni $t=15, P<0.001$, respectively), as compared to untreated cells. In untreated S2 cells, $\sim 70 \%$ of $12 a 5^{\prime}$ cons mut transcripts contained exon 12 a compared to $\sim 30 \%$ for $w t$ transcripts, but in combination with CPT treatment $\sim 100 \%$ of 12 a $5^{\prime}$ cons mut transcripts contained exon $12 \mathrm{a}$ compared to $70 \%$ for $w t$ transcripts. These data indicate that exon $12 \mathrm{a}$ inclusion occurs through at least two mechanisms: a splice site recognition-dependent mechanism that may be induced by DNA damage (as demonstrated by the increase in exon $12 \mathrm{a}$ inclusion with $12 \mathrm{a} 5^{\prime}$ cons $\mathrm{mut}$ ) and a splice site recognition-independent mechanism that can be induced by DNA damage (as demonstrated by the CPT-induced increase in exon 12a inclusion with 12a $5^{\prime}$ cons mut).

\section{A consensus TAF1 exon 12a $3^{\prime}$ splice site does not fully bypass CPT-induced exon 12a inclusion}

To examine the generality of the alternative splicing mechanism inferred from analysis of exon 13a, we examined the TAF1 exon 12a $3^{\prime}$ splice site. Unlike the exon 13a $3^{\prime}$ splice site that is weak, the exon $12 \mathrm{a} 3^{\prime}$ splice site is moderate (Table 1). We mutated the TAF1 exon 12a $3^{\prime}$ splice site to the consensus sequence ( $12 a 3^{\prime}$ cons mut) (Fig. 8A). Cells transfected with $12 a 3^{\prime}$ cons mut expressed significantly less miniTAF1-1 $(F=320, P<0.0001)$ and miniTAF1-3 $(F=31, P=0.002)$ but more miniTAF1-2 $(F=$ 210, $P<0.0001)$ and miniTAF1-4 $(F=130, P<0.0001)$ than those transfected with $w t$ miniTAF1 (Fig. 8B-D). In addition, CPT treatment significantly down-regulated 
A

B
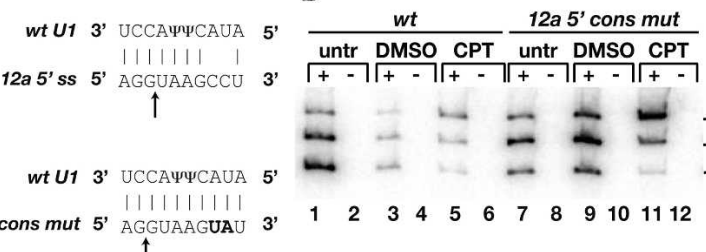
untr DMSO CPT untr DMSO CPT

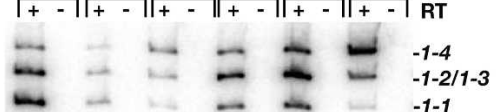

$\begin{array}{llllllllllll}1 & 2 & 3 & 4 & 5 & 6 & 7 & 8 & 9 & 10 & 11 & 12\end{array}$

$\uparrow$

C
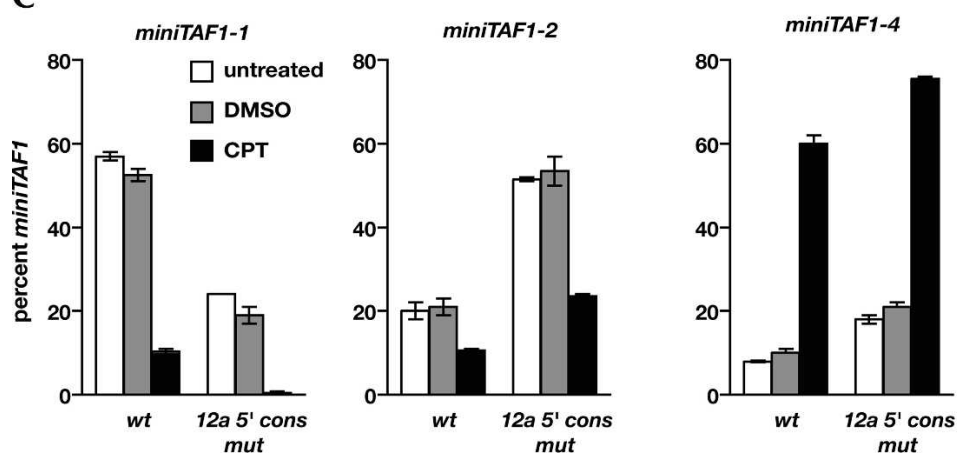

D

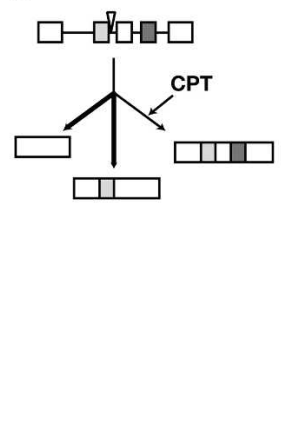

FIGURE 7. Investigation of the role of the TAF1 exon 12a 5' splice site in CPT-induced alternative splicing. (A) Shown is base pairing between the exon 12a 5' splice site (ss) in miniTAF1 pre-mRNAs and U1 snRNA. Expected base pairs are depicted as vertical lines. Pseudouridines in U1 snRNA are depicted as $\psi$. Arrows indicate splice sites and bold typeface indicates mutated nucleotides. (B) Analysis of miniTAF1 expression by RT-PCR. On the left is analysis of all four mRNA isoforms, and on the right is analysis of exon 13a-containing mRNA isoforms. Samples were analyzed from untreated (untr) S2 cells (lanes 1,2,7,8), DMSO-treated S2 cells (lanes 3,4,9,10), or CPT-treated S2 cells (lanes 5,6,11,12). Even numbered lanes contained RT-PCR samples in which reverse transcriptase (RT) was left out as a control for plasmid contamination. The transfected plasmid is indicated at the top. Positions of the miniTAF1 mRNA isoforms are shown to the right of each panel. (C) Quantitation of miniTAF1 mRNA isoforms from panel $B(n=2)$. Error bars represent the standard errors of the mean. $(D)$ A summary of the effect of CPTinduced signaling on miniTAF1 alternative splicing in the context of the consensus exon 12a $5^{\prime}$ splice site (12a $5^{\prime}$ cons mut). Shaded boxes indicate alternative exons $12 \mathrm{a}$ and $13 \mathrm{a}$. The open arrowhead indicates the mutated exon $12 \mathrm{a} 5^{\prime}$ splice site.

both wt and 12a $3^{\prime}$ cons mut miniTAF1-1 (Bonferroni $t=$ 19, $P<0.001$ and Bonferroni $t=11, P<0.001$, respectively), as compared to untreated cells. In untreated S2 cells, $\sim 70 \%$ of $12 a 3^{\prime}$ cons mut transcripts contained exon 12 a compared to $\sim 30 \%$ for $w t$ transcripts, but in CPTtreated cells $\sim 100 \%$ of $12 a 3^{\prime}$ cons mut transcripts contained exon 12 a compared to $70 \%$ for $w t$ transcripts. These data indicate that exon $12 \mathrm{a}$ inclusion occurs through at least two mechanisms: a splice site recognition-dependent mechanism (as demonstrated by the increase in exon 12 a inclusion with $12 a 3^{\prime}$ cons mut) and a splice site recognition-independent mechanism that can be induced by DNA damage signaling (as demonstrated by the CPTinduced increase in exon $12 \mathrm{a}$ inclusion with $12 a 3^{\prime}$ cons mut).

\section{IE-A is an ISS for TAF1 exon 12a inclusion}

To further investigate the regulation of exon 12a inclusion, we examined $I E-A$ and $I E-B$ because of their proximity to exon 12 a. Analysis of miniTAF1 that contained mutations in $I E-B$ revealed that $I E-B$ did not affect miniTAF1 alternative splicing (data not shown). In contrast, analysis of miniTAF1 that contained mutations of four evolutionarily conserved cytidines to guanosines (IE-A mut) revealed a role for $I E-A$ in exon 12 a inclusion (Fig. 9A). Cells transfected with IE-A mut expressed significantly less
miniTAF1-1 and miniTAF1-3 $(F=99, P=0.0002$ and $F=9.3, P=0.03$, respectively) relative to cells transfected with wt miniTAF1 (Fig. 9B-D). Mutation of IE-A upregulated the two isoforms that include exon $12 \mathrm{a}$, miniTAF1-2 and miniTAF1-4 $(F=66, P=0.0005$ and $F=22$, $P=0.0054$, respectively). Because CPT-induced signaling derepresses exon 12a inclusion more fully in the presence of an $I E-A$ mutation, $I E-A$ may be a target of CPT-induced signaling. Overall, these results indicate that $I E-A$ functions as an ISS that represses exon $12 \mathrm{a}$ inclusion despite its moderate and strong splice sites.

\section{A consensus TAF1 exon $133^{\prime}$ splice site enhances exon $12 \mathrm{a}$ exclusion}

Differences between the behavior of TAF1 exon 12a and 13a splice site consensus mutants (i.e., consensus splice sites were sufficient for exon 13a inclusion but not exon 12a inclusion) suggested that the strength of partnering splice sites affects inclusion of these exons. To examine the extent to which the weak exon $133^{\prime}$ splice site contributes to the different mechanisms of alternative splicing, we mutated the exon $133^{\prime}$ splice site to the consensus sequence (13 $3^{\prime}$ cons mut) (Fig. 10A). While the effects of this mutant were subtler than the effects of the other mutants, they were repeatable, statistically significant, and disproportionately 


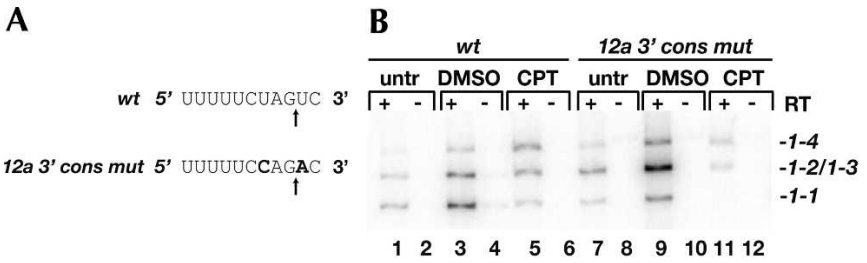

C
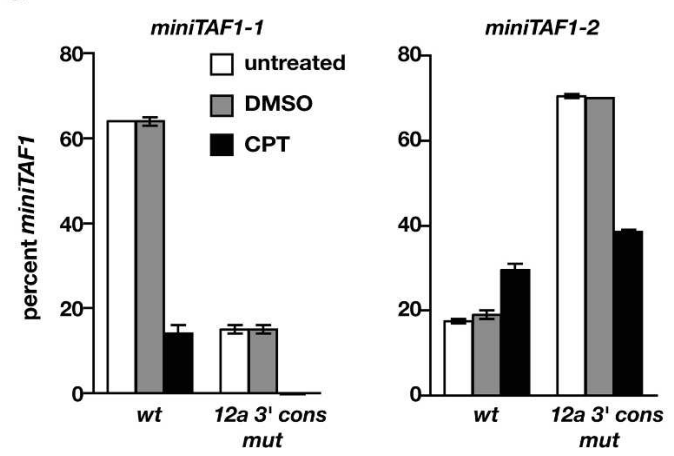

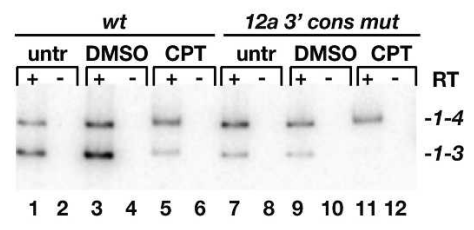

D
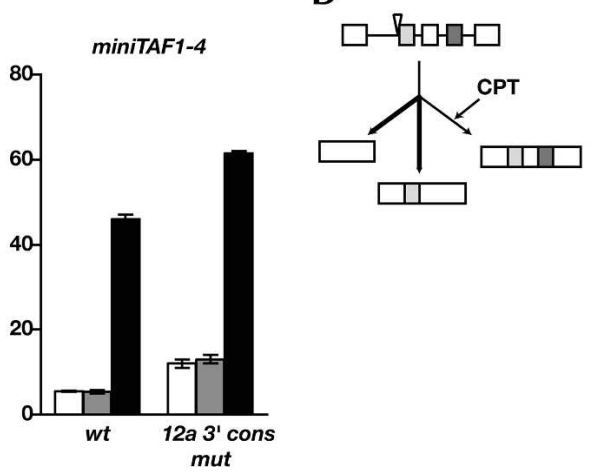

FIGURE 8. Investigation of the role of the TAF1 exon 12a 3' splice site in CPT-induced alternative splicing. (A) A comparison of $w t$ and mutant (12a 3' cons mut) exon 12a 3' splice site sequences. Arrows indicate the splice sites and bold typeface indicates mutated nucleotides. (B) Analysis of miniTAF1 expression by RT-PCR. On the left is analysis of all four mRNA isoforms, and on the right is analysis of exon 13a-containing mRNA isoforms. Samples were analyzed from untreated (untr) S2 cells (lanes 1,2,7,8), DMSO-treated S2 cells (lanes 3,4,9,10), or CPT-treated S2 cells (lanes 5,6,11,12). Even numbered lanes contained RT-PCR samples in which reverse transcriptase (RT) was left out as a control for plasmid contamination. The transfected plasmid is indicated at the top. Positions of the miniTAF1 mRNA isoforms are shown to the right of each panel. (C) Quantitation of miniTAF1 mRNA isoforms from panel $B(n=2)$. Error bars represent the standard errors of the mean. $(D)$ A summary of the effect of CPT-induced signaling on miniTAF1 alternative splicing in the context of the consensus exon 12a 3'splice site (12a 3' cons mut). Shaded boxes indicate alternative exons 12a and 13a. The open arrowhead indicates the mutated exon 12a $3^{\prime}$ splice site.

affected CPT-induced alternative splicing (Fig. 10B, cf. lanes 5 and 11). Cells transfected with $133^{\prime}$ cons mut expressed significantly more miniTAF1-1 $(F=24, P=$ $0.0028)$ but less miniTAF1-2 $(F=13, P=0.0115)$ than cells transfected with $w t$ miniTAF1 (Fig. 10B-D). CPT treatment did not affect $w t$ miniTAF1-3 (Bonferroni $t=1.2$, $P>0.05)$ but up-regulated $133^{\prime}$ cons mut miniTAF1-3 (Bonferroni $t=4.7, P<0.01$ ) and both $w t$ and $133^{\prime}$ cons mut miniTAF1-4 (Bonferroni $t=13, P<0.001$ and Bonferroni $t=8.6, P<0.001$, respectively), as compared to untreated cells. However, CPT-induced up-regulation of miniTAF1-4 was greater with wt miniTAF1 than $133^{\prime}$ cons mut (Bonferroni $t=5.1, P<0.01$ ). These data indicate that a strong exon $133^{\prime}$ splice site interferes with the CPTinduced mechanism of exon 12a inclusion, possibly by promoting pairing with the exon $125^{\prime}$ splice site. In other words, a weak exon $133^{\prime}$ splice site allows exon 12a inclusion in response to CPT-induced signals.

\section{DISCUSSION}

Here, we examined the extent to which TAF1 pre-mRNA sequences dictate changes in TAF1 alternative splicing that occur in response to CPT-induced DNA damage signals. To assess the importance of splice site strength and auxiliary elements in the context of a nascent RNA polymerase II transcript and in the presence of alternative splicing regulatory factors, we examined splicing in vivo using a TAF1 minigene that was transcribed from the endogenous TAF1 promoter.

Our results provide evidence for independent regulation of TAF1 alternative exon 12a and 13a inclusion. Signaldependent exon 12a inclusion occurred in the context of constitutive exon 13a inclusion and vice versa (Figs. 4, 5, 7, 8). Additionally, signal-dependent exon 13a inclusion appears to occur by an activation mechanism, whereas signal-dependent exon 12a inclusion appears to occur by a depression mechanism (Figs. 6, 9). Our data suggest that recognition of either weak exon 13a splice site by splicing complexes activates exon 13a inclusion and that this process is facilitated by CPT-induced signals through the ISE IE-C (Fig. 11). Similarly, our data suggest that recognition of moderate-strong exon 12a splice sites by splicing complexes activates exon 12a inclusion, but in contrast to exon 13a, this process is derepressed by CPT-induced signals through the ISS IE-A.

\section{Intronic regulatory elements can be identified by evolutionary conservation}

Alternative exons can be computationally identified with $\sim 40 \%$ accuracy solely by high exonic and flanking intronic sequence conservation between $D$. melanogaster and D. pseudoobscura (Philipps et al. 2004). Here, we extend 


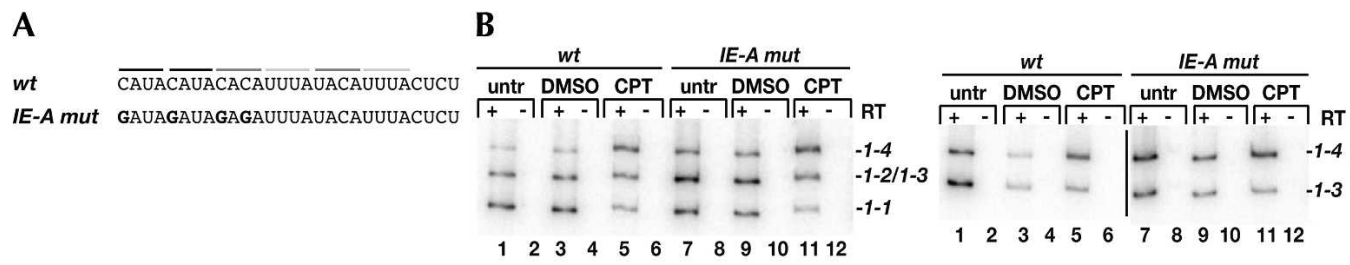

C
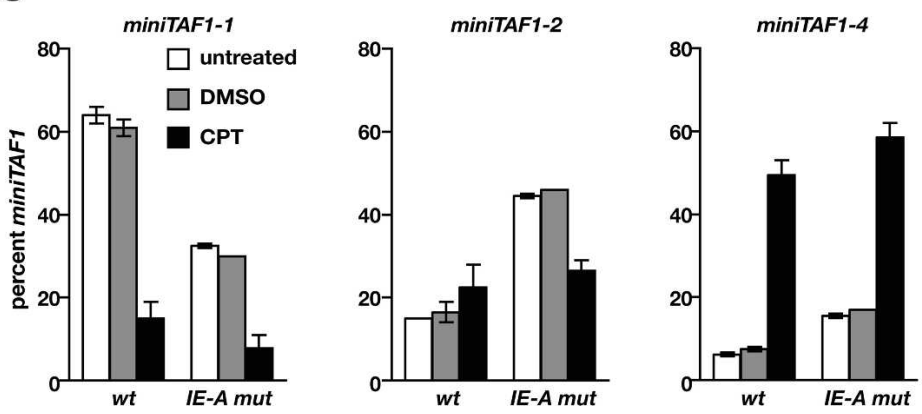

D

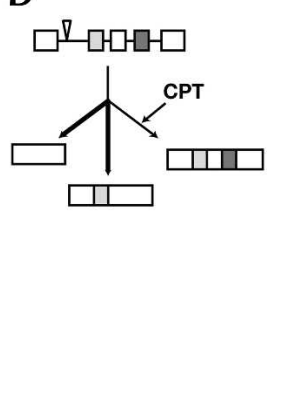

FIGURE 9. Investigation of the role of IE- $A$ in CPT-induced-alternative splicing. (A) A comparison of $w t$ and mutant $I E-A$ (IE-A mut) sequences. Mutated nucleotides are indicated in bold typeface. Lines over codons indicate the tandem repeat sequence with the boldness of the line indicating the degree of match to the most common repeat sequence. (B) Analysis of miniTAF1 expression by RT-PCR. On the left is analysis of all four mRNA isoforms, and on the right is analysis of exon 13a-containing mRNA isoforms. Samples were analyzed from untreated (untr) S2 cells (lanes 1,2,7,8), DMSO-treated S2 cells (lanes 3,4,9,10), or CPT-treated S2 cells (lanes 5,6,11,12). Even numbered lanes contain RT-PCR samples in which reverse transcriptase (RT) was left out as a control for plasmid contamination. The transfected plasmid is indicated at the top. Positions of the miniTAF1 mRNA isoforms are shown to the right of each panel. In the right panel, lanes 1-6 and 7-12 were from different gels, as indicated by the black bar. (C) Quantitation of miniTAF1 mRNA isoforms from panel $B(n=2-4)$. Error bars represent the standard errors of the mean. $(D)$ A summary of the effect of CPT-induced signaling on miniTAF1 alternative splicing in the context of the IE-A mutation. Shaded boxes indicate alternative exons $12 \mathrm{a}$ and $13 \mathrm{a}$. The open arrowhead indicates mutated $I E-A$.

such analysis to conserved sequences throughout an intron. The alignment of 12 Drosophila genomes greatly increases the comparative power of such analyses (Drosophila 12 Genomes Consortium 2007). We show that conserved repetitive intronic elements $I E-A$ and $I E-C$ are regulatory elements for TAF1 alternative splicing (Figs. 6, 9). These data provide an empirical foundation for high-throughput computational identification of intronic elements that regulate alternative splicing in Drosophila. In addition, the observation that $a b l$ alternative exon inclusion was upregulated in response to $\mathrm{CPT}$ with kinetics similar to that of endogenous TAF1 alternative exon inclusion indicates that identified intronic elements may reveal networks of coordinated, signal-regulated alternative splicing (Fig. 6E; Katzenberger et al. 2006).

\section{Different mechanisms regulate TAF1 alternative splicing in response to different DNA damage signaling pathways}

Previously, we found that CPT or IR up-regulated endogenous TAF1-4 splicing (Katzenberger et al. 2006); in contrast, only CPT up-regulated miniTAF1-4 (Figs. 2, 3). This result suggests that CPT- and IR-induced signaling pathways up-regulate TAF1-4 splicing through different mechanisms. Possibly, IR-induced TAF1-4 splicing requires an endogenous chromatin context that is not mimicked by plasmid-borne miniTAF1. Alternatively, IR-induced TAF14 splicing may require exon or intron sequences that are deleted in miniTAF1. More specifically, exon 12 in the endogenous TAF1 pre-mRNA is an internal exon, but exon 12 in the miniTAF1 pre-mRNA is a terminal exon, and terminal exons are thought to be defined by different molecular mechanisms than internal exons (Berget 1995). Regardless of mechanism, the difference between CPT- and IR-induced TAF1 alternative splicing, illuminated by mini$T A F 1$, indicates that DNA damage signaling pathways can bring about the same alternative splicing event through different mechanisms.

\section{Splice site strength is central to the mechanism of TAF1 alternative splicing}

Splice site strength is a leading factor controlling TAF1 alternative splicing. Mutation of weak exon $13 \mathrm{a} 5^{\prime}$ or $3^{\prime}$ splice sites to the consensus sequence converted exon 13a from being rarely to constitutively included in miniTAF1 mRNAs (Figs. 4, 5). Similarly, mutation of moderate or strong exon $12 \mathrm{a} 5^{\prime}$ or $3^{\prime}$ splices sites, respectively, to the consensus sequence significantly up-regulated exon $12 \mathrm{a}$ inclusion in miniTAF1 mRNA isoforms (Figs. 7, 8). We presume that $5^{\prime}$ and $3^{\prime}$ splice site mutations to the consensus sequence caused strong recruitment of the U1 snRNA or U2AF, respectively (Fig. 11). In support of the U1 snRNA binding mechanism, 
A

B

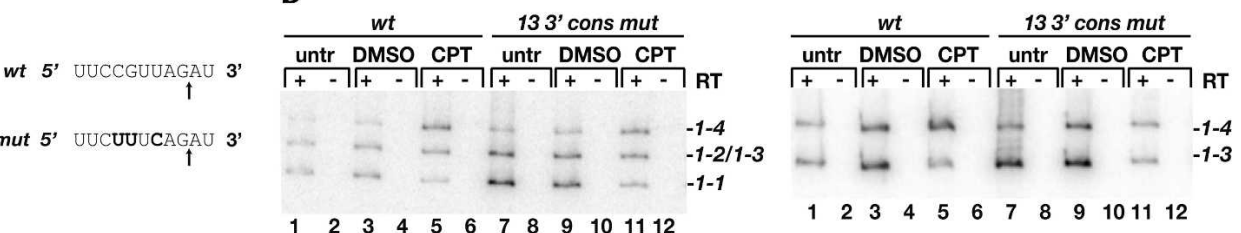

C

miniTAF1-1

$\begin{array}{llllllllllll}2 & 3 & 4 & 5 & 6 & 7 & 8 & 9 & 10 & 11 & 12\end{array}$

D
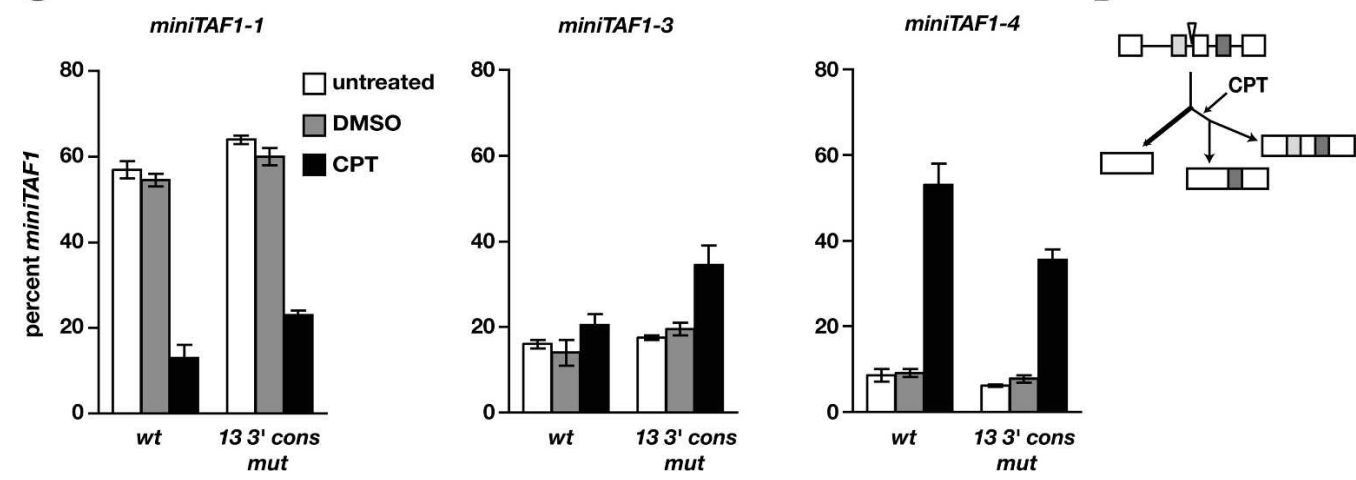

FIGURE 10. Investigation of the role of the TAF1 exon $133^{\prime}$ splice site in CPT-induced alternative splicing. (A) A comparison of $w t$ and mutant (13 3' cons mut) exon $133^{\prime}$ splice-site sequences. Arrows indicate splice sites and bold typeface indicates mutated nucleotides. (B) Analysis of miniTAF1 expression by RT-PCR. On the left is analysis of all four mRNA isoforms, and on the right is analysis of exon 13a-containing mRNA isoforms. Samples were analyzed from untreated (untr) S2 cells (lanes 1,2,7,8), DMSO-treated S2 cells (lanes 3,4,9,10), or CPT-treated S2 cells (lanes 5,6,11,12). Even numbered lanes contained RT-PCR samples in which reverse transcriptase (RT) was left out as a control for plasmid contamination. The transfected plasmid is indicated at the top. Positions of the miniTAF1 mRNA isoforms are shown to the right of each panel. (C) Quantitation of miniTAF1 mRNA isoforms from panel $B(n=2)$. Error bars represent the standard errors of the mean. $(D)$ A summary of the effect of CPT-induced signaling on miniTAF1 alternative splicing in the context of the consensus exon $133^{\prime}$ splice site (13 $3^{\prime}$ cons mut). Shaded boxes indicate alternative exons 12a and 13a. The open arrowhead indicates the mutated exon $133^{\prime}$ splice site.

expression of a $U 1$ snRNA containing a mutant $5^{\prime}$ end that was perfectly complementary to the exon 13 a $5^{\prime}$ splice site, but not one with a wild type $5^{\prime}$ end that was partially complementary to the exon 13 a $5^{\prime}$ splice site, significantly upregulated inclusion of exon 13a in endogenous TAF1 mRNAs (Fig. 4E). Thus, as demonstrated in human cells, in Drosophila cells, extended complementarity between U1 snRNA and the $5^{\prime}$ splice site increases $5^{\prime}$ splice site recognition (Freund et al. 2005). Furthermore, despite demonstrated contributions of the U1 snRNP protein U1-C to $5^{\prime}$ splice site recognition, base pairing between $U 1$ snRNA and the $5^{\prime}$ splice site is dominant in TAF1 alternative splicing ( $\mathrm{Du}$ and Rosbash 2002; Förch et al. 2002). We have not directly examined U2AF binding to TAF1 3' splice sites, but U2AF is commonly involved in $3^{\prime}$ splice site recognition and regulates alternative 3 ' splice site recognition (Merendino et al. 1999; Wu et al. 1999; Zorio and Blumenthal 1999; Pacheco et al. 2006).

\section{Studies of exons 12a and 13a illuminate different types of exon definition}

Differences between the splicing of miniTAF1 exon 12a and 13a splice site consensus mutants provided insights into the intricacies of splicing factor recruitment. Mutation of either exon 13a splice site but neither exon 12a splice site to the consensus sequence was sufficient for constitutive exon inclusion (Figs. 3, 4, 6, 7). In the case of exon 13a, this finding suggests that strong binding of $U 1$ snRNA to the $5^{\prime}$ splice site results in strong binding of U2AF to the upstream $3^{\prime}$ splice site, and strong binding of U2AF to the $3^{\prime}$ splice site results in strong binding of $U 1$ snRNA to the downstream 5' splice site (Fig. 11). This is a classic example of exon definition (Berget 1995). In exon definition, mutual stabilization of U1 snRNA and U2AF binding to $5^{\prime}$ and $3^{\prime}$ splice sites, respectively, occurs across the exon. This is expected for exon 13a since it is short (93 nt) and the upstream intron is large (324 nt) (Fig. 1A). It has been demonstrated that splice site recognition across the intron (intron definition) ceases when the intron length exceeds

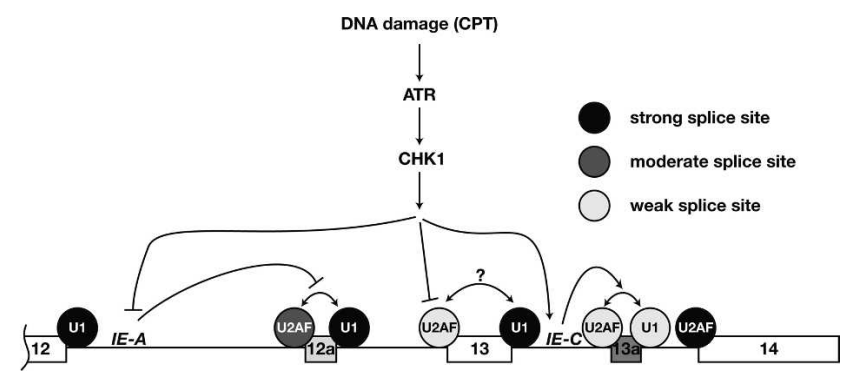

FIGURE 11. A model for the regulation of TAF1 alternative splicing by CPT-induced DNA damage signals. Lines with arrowheads on each end indicate exon definition; exon 13 is presumed to be exon defined. Details of this model are provided in the Discussion. 
200-250 nt (Fox-Walsh et al. 2005). Similarly, a consensus $5^{\prime}$ splice site up-regulated exon 12 a to a similar degree as a $3^{\prime}$ consensus splice site. However, neither mutation was sufficient to drive constitutive $12 \mathrm{a}$ inclusion and bypass CPT-induced inclusion. Differences between the splicing of miniTAF1 exon 12a and 13a splice site consensus mutants are likely not attributable to U6 snRNA binding since the mutated 5' splice sites had the same base-pairing potential with U6 snRNA. Thus, efficient recruitment of splicing factors via exon definition is not always sufficient for constitutive exon inclusion.

\section{TAF1 exon 12a inclusion may be regulated by modulation of splice site pairing}

Although the effect of the exon $133^{\prime}$ consensus splice site mutation was subtle, it supports a growing body of literature indicating that alternative splicing regulation involves modulated splice site pairing (House and Lynch 2008). RNAi-mediated depletion of general splicing factors, such as U2AF, and splicing activators in S2 cells has been shown to unexpectedly up-regulate alternative exon inclusion, including exons $12 \mathrm{a}$ and $13 \mathrm{a}$ of Drosophila TAF1 (Park et al. 2005; Katzenberger et al. 2006). Similarly, Hastings et al. (2007) found that reducing the level of the large subunit of U2AF in mammalian cells altered alternative splicing patterns. Our data support a kinetic model of alternative splicing, wherein down-regulation of general splicing factors and splicing activators reduces the efficiency of splice site pairing between constitutive exons, which allows alternative exons to compete for pairing.

Intriguingly, the exon $133^{\prime}$ splice site consensus mutant was the only splice site mutant to have a disproportionate effect specifically on CPT-induced splicing (Fig. 10). This discovery illustrates the importance of systematically studying the regulation of alternative splicing in a context as close to that of the endogenous gene as possible. Although many metazoan genes contain multiple exons that may be coordinately regulated, mutation analysis is typically done in heterologous single alternative exon constructs. While such studies are valuable for identifying elements that are sufficient for splicing regulation, single exon analysis would not have detected the effect of the exon $133^{\prime}$ splice site on exon $12 \mathrm{a}$ inclusion. The specificity of the effect for CPTinduced splicing implies that splice site pairing may be a target for DNA damage and other signaling pathways.

\section{Biological relevance of mechanistically independent TAF1 exon 12a and 13a splicing}

The CPT-induced DNA damage signal resulted in increased exon $12 \mathrm{a}$ and $13 \mathrm{a}$ inclusion for both endogenous TAF1 and miniTAF1. Studies of miniTAF1 indicate that inclusion of exons $12 \mathrm{a}$ and $13 \mathrm{a}$ does not occur by a collaborative mechanism but rather by independent mechanisms that can be induced by the same signal. Inclusion of exon $12 \mathrm{a}$ was not affected by increased inclusion of exon 13a in $13 a$ $5^{\prime}$ cons mut, and exon 13a inclusion was not affected by increased inclusion of exon 12a in $12 a 5^{\prime}$ cons mut (Figs. 3, 6). CPT induced exon 12a inclusion to the same extent with wt miniTAF1 as $13 a 5^{\prime}$ cons mut and exon 13 a inclusion to the same extent with $w t$ miniTAF1 as $12 a 5^{\prime}$ cons mut. In the context of $I E-C$ mutations, CPT-induced exon $12 \mathrm{a}$ inclusion did not require CPT-induced exon 13a inclusion (Fig. 5). Similarly, CPT-induced exon 13a inclusion did not require $\mathrm{CPT}$-induced exon $12 \mathrm{a}$ inclusion in the context of the exon 13 3' consensus splice site (Fig. 9). We hypothesize that TAF1 pre-mRNA elements identified in this study are independently targeted by developmental signaling pathways to result in tissue-specific inclusion of exon $12 \mathrm{a}$ and/or 13a, which may play a role in tissue-specific promoter regulation via altered TAF1 DNA binding properties (Metcalf and Wassarman 2006, 2007). The challenge now is to create transgenic flies in which splice sites and intronic elements are mutated to investigate the regulation and function of TAF1 alternative splicing during development.

\section{MATERIALS AND METHODS}

\section{Sequence comparisons}

Sequences of TAF1 shown in Figure 1A were retrieved from Flybase (flybase.org) and aligned by ClustalW (Higgins et al. 1994). Analysis of splice site frequency in $D$. melanogaster was performed using both database and analysis software described by Sheth et al. (2006). For $3^{\prime}$ splice site comparisons, the motif start position was 29 and the motif was $7 \mathrm{nt}$ long. For $5^{\prime}$ splice site comparisons, the motif start position was 6 and the motif was $8 \mathrm{nt}$ long.

\section{Plasmids}

The miniTAF1 plasmid was generated by cloning a sequence encoding a FLAG-epitope tag (5'-GACTACAAGGACGACGATG ACAAG-3') in-frame with a TAF1 genomic fragment encoding exons 12-14 (positions 118,598-122,168 of GenBank accession number AC095013.1) and downstream from a TAF1 genomic fragment that contained the transcription promoter (positions 110,087-112,630 of GenBank accession number AC095013.1). Green fluorescent protein (GFP) expression plasmid was generated by cloning a NcoI-SpeI fragment from $p H$-Stinger (Drosophila Genomics Resource Center; Barolo et al. 2000) into pBluescript II (Stratagene). U1 snRNA plasmid was generated by cloning a U1 $21 D$ genomic fragment (positions 64,374-65,602 of GenBank accession number AC092222) into pCRII (Invitrogen). This clone included the region previously shown to contain all of the elements necessary for U1 snRNA expression (Lo and Mount 1990). Site-directed mutagenesis of miniTAF1 and U1 snRNA was carried out using the QuikChange mutagenesis kit (Stratagene).

\section{Cell culture, transfection, and treatments}

Drosophila S2 cells were maintained at $27.5^{\circ} \mathrm{C}$ in Schneider's Drosophila medium containing $10 \%$ heat-inactivated fetal bovine 
serum, $100 \mathrm{U} / \mathrm{mL}$ penicillin, and $100 \mu \mathrm{g} / \mathrm{mL}$ of streptomycin (Invitrogen). Before transfection, cells were subcultured to $80 \%-$ $90 \%$ confluence in $2 \mathrm{~mL}$ of media. For miniTAF1 experiments, cells were transfected with $300 \mathrm{ng}$ GFP plasmid, as a transfection control, and $1 \mu \mathrm{g}$ miniTAF1 plasmid using Effectene (Qiagen). After $24 \mathrm{~h}$ at $27.5^{\circ} \mathrm{C}, \mathrm{CPT}$ (Sigma) in dimethyl sulfoxide (DMSO; Sigma) was added to cells to $20 \mu \mathrm{M}$ and incubated at $27.5^{\circ} \mathrm{C}$ overnight. Untreated and DMSO-treated cells served as negative controls. For U1 snRNA expression experiments, samples were transfected with $200 \mathrm{ng}$ GFP plasmid, $500 \mathrm{ng}$ U1 snRNA plasmid, and $800 \mathrm{ng} p C R I I$ plasmid as carrier DNA (Invitrogen). For IR treatment, transfected cells were irradiated with 80 Gy by a Mark 1 irradiator and allowed to recover at $27.5^{\circ} \mathrm{C}$ for $3 \mathrm{~h}$.

\section{Reverse transcriptase-PCR (RT-PCR)}

Total RNA was isolated from S2 cells using an RNeasy Mini kit (Qiagen). cDNA was synthesized using $1 \mu \mathrm{g}$ RNA in a $20 \mu \mathrm{L}$ RT reaction using an iScript cDNA Synthesis kit (Bio-Rad). The resulting cDNA was diluted 1:10 with $\mathrm{dd}_{2} \mathrm{O}$. Each $50 \mu \mathrm{L}$ PCR contained $10 \mu \mathrm{L}$ diluted cDNA. Reactions contained $20 \mathrm{mM}$ Tris$\mathrm{Cl}$ (pH 8.4), $50 \mathrm{mM} \mathrm{KCl,} 1.5 \mathrm{mM} \mathrm{MgCl}_{2}, 200 \mu \mathrm{M}$ dNTPs, $1.2 \mu \mathrm{M}$ each primer, $1.9 \mu \mathrm{Ci} \alpha-{ }^{32} \mathrm{P}-\mathrm{dCTP}$ (GE Healthcare), and $5 \mathrm{U}$ Taq polymerase (Invitrogen). PCR cycling conditions in the linear range were $94^{\circ} \mathrm{C}$ for $4 \mathrm{~min}$ followed by 21 cycles of $94^{\circ} \mathrm{C}$ for $1 \mathrm{~min}, 55^{\circ} \mathrm{C}$ for $1 \mathrm{~min}$, and $72^{\circ} \mathrm{C}$ for $1 \mathrm{~min}$. Reaction products were extracted with phenol:chloroform:isoamyl alcohol (24:24:1), precipitated with ammonium acetate and ethanol, fractionated by $5 \%$ PAGE, and visualized and quantitated by PhosphorImager (Molecular Dynamics). For abl PCR, a radioactive nucleotide was not used and the cycling conditions were $94^{\circ} \mathrm{C}$ for $4 \mathrm{~min}$ followed by $30 \mathrm{cycles}$ of $94^{\circ} \mathrm{C}$ for $15 \mathrm{sec}, 55^{\circ} \mathrm{C}$ for $30 \mathrm{sec}$, and $72^{\circ} \mathrm{C}$ for 30 sec. PCR products were fractioned by $1.5 \%$ agarose gel electrophoresis. Where indicated, cDNAs were cloned into $p C R I I$ (Invitrogen) and sequenced at the University of Wisconsin Biotechnology Center. Primer sets for PCR (oriented $5^{\prime}$ to $3^{\prime}$ ) were as follows: FLAG GACTACAAGGACGACGATGACAAG, TAF1 exon 14 ATCTGAGGGATCGTAGTTGG, TAF1 exon 13-exon 13a junction ATCGGCTCCTCTGCCATCTGGTG, abl forward GGTCTCAACATATTCACCAG, and $a b l$ reverse GTAAT GGACGCGACTGCAG (Integrated DNA Technologies).

\section{Quantitative real-time PCR (qPCR)}

Total RNA was isolated from S2 cells and cDNA was synthesized using $1 \mu \mathrm{g}$ RNA as described above. cDNA was diluted 1:10 with $\mathrm{ddH}_{2} \mathrm{O}$. Each $25 \mu \mathrm{L}$ qPCR contained $5 \mu \mathrm{L}$ diluted cDNA, $12.5 \mu \mathrm{L}$ iQ SYBR Green Supermix (Bio-Rad), and $500 \mathrm{nM}$ each primer. Reactions were carried out using an iCycler Thermal Cycler (BioRad). PCR cycling conditions were $95^{\circ} \mathrm{C}$ for $3 \mathrm{~min}$ followed by cycles of $95^{\circ} \mathrm{C}$ for $30 \mathrm{sec}, 61^{\circ} \mathrm{C}$ for $30 \mathrm{sec}$, and $72^{\circ} \mathrm{C}$ for $30 \mathrm{sec}$. Melt curve analysis was carried out to evaluate the homogeneity of the reaction products. Amplicons were 75-270 bp long. Primer sets for qPCR (oriented $5^{\prime}$ to $3^{\prime}$ ) were as follows: actin CGAAGA AGTTGCTGCTCTGGTTGTCG and GGACGTCCCACAATCGA TGGGAAG, total TAF1 GGCCAAGTCAAATGATGCATCTAGT CCC and CAGCTTCCGATCCGCATCCTTTG, TAF1-3 CTCAAC TGCACCATTGCTTCGGCC and CGTGGAGGAGGATCTCCAA TGCTC, TAF1-4 CTGGATGAAGATCTCCAATGCTCCAC and CGATCGGCTCCTCTGCCATCTG.

\section{Quantitation and statistics}

MiniTAF1 isoforms were quantitated on a PhosphorImager (Molecular Dynamics). Isoform levels were corrected for cytidine content and normalized to total miniTAF1 signal. The following equations were used to determine miniTAF1-2 and miniTAF1-3 levels:

$$
\begin{aligned}
(\text { miniTAF1-3 })_{F 14} & =\frac{(\text { miniTAF1-3 })_{F 13 a}}{(\text { miniTAF1-4 })_{F 13 a}} \times(\text { miniTAF1-4 })_{F 14}, \\
(\text { miniTAF1-2 })_{F 14} & =(\text { miniTAF1-2/1-3 })_{F 14}-(\text { miniTAF1-3 })_{F 14}
\end{aligned}
$$

The F14 subscript refers to the normalized signal from the PCR using the FLAG and exon 14 primers, the F13a subscript refers to the normalized signal from the PCR using the FLAG and exon 13a primers, and (miniTAF1-2/1-3) F14 $_{14}$ refers to the band from the PCR using the FLAG and exon 14 primers in which miniTAF1-2 and miniTAF1-3 comigrated. For each isoform in each experiment, a two-way analysis of variance (ANOVA) was performed in conjunction with Bonferroni post-tests using Prism 4.0c (Graphpad). Samples from separately transfected wells of cells were used as independent replicates. For qPCR assays, the cDNA of interest was quantitated relative to actin by the formula $\left(\mathrm{E}_{\text {actin }}{ }^{\mathrm{Ct}(\mathrm{actin})}\right)$ / $\left(\mathrm{E}_{\text {target }} \mathrm{Ct}(\right.$ target)$)$ (Pfaffl 2001). $E$ is an empirically derived PCR efficiency factor, and $C t$ is the threshold value for amplification. cDNA levels were normalized to the level from an independent well of cells. Samples from separate wells of cells were used as independent replicates. T-tests were performed using Prism 4.0c (Graphpad).

\section{ACKNOWLEDGMENTS}

We thank R. Katzenberger, C. Metcalf, S. Rimkus, and D. Brow for helpful comments throughout the course of these studies and $\mathrm{P} . \mathrm{Vu}$ for conducting the analysis of TAF1 splicing in D. virilis. This work was supported by National Science Foundation grant MCB-0614059 (to D.A.W.) and by pre-doctoral fellowships from NIH training grant T32 GM08688 and the PhRMA Foundation (to M.S.M.).

Received February 27, 2008; accepted May 6, 2008.

\section{REFERENCES}

Abraham, R.T. 2001. Cell cycle checkpoint signaling through the ATM and ATR kinases. Genes \& Dev. 15: 2177-2196.

Barolo, S., Carver, L.A., and Posakony, J.W. 2000. GFP and $\beta$ galactosidase transformation vectors for promoter/enhancer analysis in Drosophila. Biotechniques 29: 726-732.

Berget, S.M. 1995. Exon recognition in vertebrate splicing. J. Biol. Chem. 270: 2411-2414.

Black, D.L. 2003. Mechanisms of alternative pre-messenger RNA splicing. Annu. Rev. Biochem. 72: 291-336.

Brow, D.A. 2002. Allosteric cascade of spliceosome activation. Annu. Rev. Genet. 36: 333-360.

Cuccurese, M., Russo, G., Russo, A., and Pietropaolo, C. 2005. Alternative splicing and nonsense-mediated decay regulate mammalian ribosomal gene expression. Nucleic Acids Res. 33: 5965-5977.

Drosophila 12 Genomes Consortium 2007. Evolution of genes and genomes on the Drosophila phylogeny. Nature 450: 203-218.

$\mathrm{Du}, \mathrm{H}$. and Rosbash, M. 2002. The U1 snRNP protein U1C recognizes the $5^{\prime}$ splice site in the absence of base pairing. Nature 419: $86-90$. 
Förch, P., Puig, O., Martínez, C., Séraphin, B., and Valcárcel, J. 2002. The splicing regulator TIA-1 interacts with U1-C to promote U1 snRNP binding to 5' splice sites. EMBO J. 21: 6882-6892.

Fox-Walsh, K.L., Dou, Y., Lam, B.J., Hung, S., Baldi, P.F., and Hertel, K.J. 2005. The architecture of pre-mRNAs affects mechanism of splice-site pairing. Proc. Natl. Acad. Sci. 102: 16176-16181.

Freund, M., Asang, C., Kammler, S., Konermann, C., Krummheuer, J., Hipp, M., Meyer, I., Gierling, W., Theiss, S., Preuss, T., et al. 2003. A novel approach to describe a U1 snRNA binding site. Nucleic Acids Res. 31: 6963-6975.

Freund, M., Hicks, M.J., Konermann, C., Otte, M., Hertel, K.J., and Schaal, H. 2005. Extended base pair complementarity between U1 and the 5' splice site does not inhibit splicing in higher eukaryotes, but rather increases $5^{\prime}$ splice site recognition. Nucleic Acids Res. 33: 5112-5119.

Goldstrohm, A.C., Greenleaf, A.L., and Garcia-Blanco, M.A. 2001. Cotranscriptional splicing of pre-messenger RNAs: Considerations for the mechanism of alternative splicing. Gene 277: 31-47.

Graveley, B.R. 2001. Alternative splicing: Increasing diversity in the proteomic world. Trends Genet. 17: 100-107.

Hastings, M.L., Allemand, E., Duelli, D.M., Myers, M.P., and Krainer, A.R. 2007. Control of pre-mRNA splicing by the general splicing factors PUF60 and U2AF65. PLoS ONE 2: e538. doi: 10.1371/journal.pone.0000538.

Henkemeyer, M.J., Bennett, R.L., Gertler, F.G., and Hoffmann, F.M. 1988. DNA sequence, structure, and tyrosine kinase activity of the Drosophila melanogaster Abelson proto-oncogene homolog. Mol. Cell. Biol. 8: 843-853.

Higgins, D., Thompson, J., Gibson, T., Thompson, J.D., Higgins, D.G., and Gibson, T.J. 1994. CLUSTAL W: Improving the sensitivity of progressive multiple sequence alignment through sequence weighting, position-specific gap penalties and weight matrix choice. Nucleic Acids Res. 22: 4673-4680.

House, A.E. and Lynch, K.W. 2008. Regulation of alternative splicing: More than just the ABCs. J. Biol. Chem. 283: 1217-1221.

Itoh, H., Washio, T., and Tomita, M. 2004. Computational comparative analyses of alternative splicing regulation using full-length cDNA of various eukaryotes. RNA 10: 1005-1018.

Izquierdo, J.M., Majós, N., Bonnal, S., Martinez, C., Castelo, R., Guigó, R., Bilbao, D., and Valcárcel, J. 2005. Regulation of Fas alternative splicing by antagonistic effects of TIA-1 and PTB on exon definition. Mol. Cell 19: 475-484.

Kandels-Lewis, S. and Séraphin, B. 1993. Involvement of U6 snRNA in $5^{\prime}$ splice site selection. Science 262: 2035-2039.

Katzenberger, R.J., Marengo, M.S., and Wassarman, D.A. 2006. ATM and ATR pathways signal alternative splicing of Drosophila TAF1 pre-mRNA in response to DNA damage. Mol. Cell. Biol. 26: $9256-$ 9267.

Kornblihtt, A.R. 2005. Promoter usage and alternative splicing. Curr. Opin. Cell Biol. 17: 262-268.

Lesser, C.F. and Guthrie, C. 1993. Mutations in U6 snRNA that alter splice site specificity: Implications for the active site. Science 262: 1982-1988.

Lo, P.C. and Mount, S.M. 1990. Drosophila melanogaster genes for U1 snRNA variants and their expression during development. Nucleic Acids Res. 18: 6971-6979.

Merendino, L., Guth, S., Bilbao, D., Martínez, C., and Valcárcel, J. 1999. Inhibition of msl-2 splicing by Sex-lethal reveals interaction between U2AF35 and the $3^{\prime}$ splice site AG. Nature 402: 838-841.

Metcalf, C.E. and Wassarman, D.A. 2006. DNA binding properties of TAF1 isoforms with two AT-hooks. J. Biol. Chem. 281: 30015-30023.

Metcalf, C.E. and Wassarman, D.A. 2007. Nucleolar colocalization of TAF1 and testis-specific TAFs during Drosophila spermatogenesis. Dev. Dyn. 236: 2836-2843.

Pacheco, T.T., Coelho, M.B., Desterro, J.M.P., Mollet, I., and CarmoFonseca, M. 2006. In vivo requirement of the small subunit of
U2AF for recognition of a weak $3^{\prime}$ splice site. Mol. Cell. Biol. 26: 8183-8190.

Park, J.W., Parisky, K., Celotto, A.M., Reenan, R.A., and Graveley, B.R. 2005. Identification of alternative splicing regulators by RNA interference in Drosophila. Proc. Natl. Acad. Sci. 101: 15974-15979.

Patel, A.A. and Steitz, J.A. 2003. Splicing double: Insights from the second spliceosome. Nat. Rev. Mol. Cell Biol. 4: 960-970.

Pfaffl, M.W. 2001. A new mathematical model for relative quantification in real-time RT-PCR. Nucleic Acids Res. 29: e45. doi: 10.1093/nar/29.9.e45.

Philipps, D.L., Park, J.W., and Graveley, B.R. 2004. A computational and experimental approach toward a priori identification of alternatively spliced exons. RNA 10: 1838-1844.

Ram, O. and Ast, G. 2003. SR proteins: A foothold on the exon before the transition from intron to exon definition. Trends Genet. 23: 5-7.

Rothrock, C.R., House, A.E., and Lynch, K.W. 2005. HnRNP L represses exon splicing via a regulated exonic splicing silencer. EMBO J. 24: 2792-2802.

Ruskin, B., Zamore, P.D., and Green, M.R. 1988. A factor, U2AF, is required for U2 snRNP binding and splicing complex assembly. Cell 52: 207-219.

Schawaroch, V. 2002. Phylogeny of a paradigm lineage: The Drosophila melanogaster species group. Biol. J. Linn. Soc. Lond. 76: 21-37.

Schwerk, C. and Schulze-Osthoff, K. 2005. Regulation of apoptosis by alternative pre-mRNA splicing. Mol. Cell 19: 1-13.

Séraphin, B., Kretzner, L., and Rosbash, M. 1988. A U1 snRNA:premRNA base pairing interaction is required early in yeast spliceosome assembly but does not uniquely define the $5^{\prime}$ cleavage site. EMBO J. 7: 2533-2538.

Sheth, N., Roca, X., Hastings, M.L., Roeder, T., Krainer, A.R., and Sachidanandam, R. 2006. Comprehensive splice-site analysis using comparative genomics. Nucleic Acids Res. 34: 3955-3967.

Shin, C. and Manley, J.L. 2004. Cell signalling and the control of premRNA splicing. Nat. Rev. Mol. Cell Biol. 5: 727-738.

Siliciano, P.G. and Guthrie, C. 1988. 5' splice site selection in yeast: Genetic alterations in base-pairing with $\mathrm{U} 1$ reveal additional requirements. Genes \& Dev. 2: 1258-1267.

Stamm, S. 2002. Signals and their transduction pathways regulating alternative splicing: A new dimension of the human genome. Hum. Mol. Genet. 11: 2409-2416.

Truong, T., Sun, G., Doorly, M., Wang, J.Y.L., and Schwartz, M.A. 2003. Modulation of DNA damage-induced apoptosis by cell adhesion is independently mediated by p53 and c-Abl. Proc. Natl. Acad. Sci. 100: 10281-10286.

Wassarman, D.A. and Steitz, J.A. 1992. Interactions of small nuclear RNAs with precursor messenger RNA during in vitro splicing. Science 257: 1918-1925.

Weinzierl, R.O., Dynlacht, B.D., and Tjian, R. 1993. Largest subunit of Drosophila transcription factor IID directs assembly of a complex containing TBP and a coactivator. Nature 362: 511-517.

Weir, M. and Rice, M. 2004. Ordered partitioning reveals extended splice-site consensus information. Genome Res. 14: 67-78.

Wu, S., Romfo, C.M., Nilson, T.W., and Green, M.R. 1999. Functional recognition of the $3^{\prime}$ splice site AG by the splicing factor U2AF35. Nature 402: 832-835.

Zamore, P.D. and Green, M.R. 1989. Identification, purification, and biochemical characterization of U2 small nuclear ribonucleoprotein auxiliary factor. Proc. Natl. Acad. Sci. 86: 9243-9247.

Zamore, P.D., Patton, J.G., and Green, M.R. 1992. Cloning and domain structure of the mammalian splicing factor U2AF. Nature 355: 609-614.

Zhuang, Y. and Weiner, A.M. 1986. Compensatory base change in Ul snRNA suppresses a $5^{\prime}$ splice site mutation. Cell 46: 827-835.

Zorio, D.A. and Blumenthal, T. 1999. Both subunits of U2AF recognize the $3^{\prime}$ splice site in Caenorhabditis elegans. Nature 402: 835-838. 

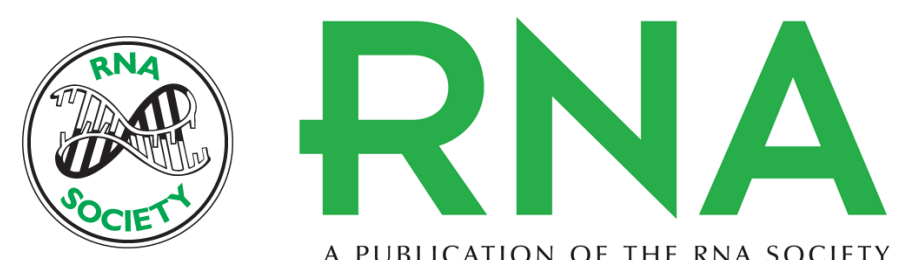

A PUBLICATION OF THE RNA SOCIETY

\section{A DNA damage signal activates and derepresses exon inclusion in Drosophila TAF1 alternative splicing}

Matthew S. Marengo and David A. Wassarman

RNA 2008 14: 1681-1695

References This article cites 52 articles, 19 of which can be accessed free at: http://rnajournal.cshlp.org/content/14/8/1681.full.html\#ref-list-1

License

Email Alerting Receive free email alerts when new articles cite this article - sign up in the box at the Service top right corner of the article or click here. 